\title{
Investigating Extreme Dependences: Concepts and Tools *
}

\author{
Y. Malevergne $e^{1,2}$ and D. Sornette ${ }^{1,3}$ \\ ${ }^{1}$ Laboratoire de Physique de la Matière Condensée CNRS UMR 6622 \\ Université de Nice-Sophia Antipolis, 06108 Nice Cedex 2, France \\ ${ }^{2}$ Institut de Science Financière et d'Assurances - Université Lyon I \\ 43, Bd du 11 Novembre 1918, 69622 Villeurbanne Cedex \\ ${ }^{3}$ Institute of Geophysics and Planetary Physics and Department of Earth and Space Science \\ University of California, Los Angeles, California 90095, USA \\ email: Yannick.Malevergne@unice.fr and sornette@unice.fr \\ fax: (33) 492076754
}

October 29, 2018

\begin{abstract}
We investigate the relative information content of six measures of dependence between two random variables $X$ and $Y$ for large or extreme events for several models of interest for financial time series. The six measures of dependence are respectively the linear correlation $\rho_{v}^{+}$and Spearman's rho $\rho_{s}(v)$ conditioned on signed exceedance of one variable above the threshold $v$, or on both variables $\left(\rho_{u}\right)$, the linear correlation $\rho_{v}^{s}$ conditioned on absolute value exceedance (or large volatility) of one variable, the so-called asymptotic tail-dependence $\lambda$ and a probability-weighted tail dependence coefficient $\bar{\lambda}$. The models are the bivariate Gaussian distribution, the bivariate Student's distribution, and the factor model for various distributions of the factor. We offer explicit analytical formulas as well as numerical estimations for these six measures of dependence in the limit where $v$ and $u$ go to infinity. This provides a quantitative proof that conditioning on exceedance leads to conditional correlation coefficients that may be very different from the unconditional correlation and gives a straightforward mechanism for fluctuations or changes of correlations, based on fluctuations of volatility or changes of trends. Moreover, these various measures of dependence exhibit different and sometimes opposite behaviors, suggesting that, somewhat similarly to risks whose adequate characterization requires an extension beyond the restricted one-dimensional measure in terms of the variance (volatility) to include all higher order cumulants or more generally the knowledge of the full distribution, tail-dependence has also a multidimensional character.
\end{abstract}

\section{Introduction}

The Oct. 19, 1987, stock-market crash stunned Wall Street professionals, hacked about $\$ 1$ trillion off the value of all U.S. stocks, and elicited predictions of another Great Depression. On "Black Monday," the Dow Jones industrial average plummeted 508 points, or 22.6 percent, to $1,738.74$. It was both the largest one-day point and percentage loss ever for the blue-chip index until the large loss of 684.81 points due to the events

\footnotetext{
${ }^{*}$ We acknowledge helpful discussions and exchanges with J.P. Laurent, F. Lindskog and V. Pisarenko. This work was partially supported by the James S. Mc Donnell Foundation 21st century scientist award/studying complex system.
} 
on September 11, 2001. The broader markets followed the Dow downward. The S\&P 500 index lost more than 20 percent, falling 57.86 to 224.84. The Nasdaq Composite index dived 46.12 to 360.21. No Dow components emerged unscathed from Black Monday. Even market stalwarts suffered massive share losses. IBM shed $31-3 / 4$ to close at $103-1 / 3$, while USX lost $12-1 / 2$ to $21-1 / 2$ and Eastman Kodak fell $27-1 / 4$ to $62-7 / 8$. The crash splattered technology stocks as well. On the Nasdaq, Apple Computer lost $11-3 / 4$ to close at $36-1 / 2$, while Intel dropped 10 to 42 . Stocks descended quickly on Black Monday, with the Dow falling 200 points soon after the opening bell to trade at around 2,046. Yet by 10 a.m., the index had crept back up above 2,100, beginning a pattern of rebound and retreat that would continue for most of the day. Later, with 75 minutes left in the trading day, it looked like the Dow would escape with a loss of "only" about 200 points. But the worst was yet to come. Starting at about 2:45 p.m., a massive sell-off began, eventually ripping 300 more points off of the Dow. At the closing bell, the Dow appeared to have suffered an amazing loss of about 400 points. However, heavy volume kept the New York Stock Exchange's computers running hours behind trading. Only about two hours later would investors realize that the day's total loss exceeded 500 points.

This event epitomizes the observation often reported by market professionals that, "during major market events, correlations change dramatically" [Bookstaber (1997)]. The possible existence of changes of correlation, or more precisely of changes of dependence, between assets in different market phases has obvious implications in risk assessment, portfolio management and in the way policy and regulation should be performed.

There are two distinct classes of mechanisms for understanding "changes of correlations", not necessarily mutually exclusive.

- It is possible that there are genuine changes with time of the unconditional correlations and thus of the underlying structure of the dynamical processes, as observed by identifying shifts in ARMAARCH/GARCH processes [Silvapulle and Granger (2001)], in regime-switching models [Ang and Bekaert (2000), Ang and Chen (2001)] or in contagion models [Quintos (2001), Quintos et al. (2001)]. [Longin and Solnik (1995), Tsui and Yu (1999)] and many others have shown that the hypothesis of a constant conditional correlation for stock returns or international equity returns must be rejected. In fact, there is strong evidence that the correlations are not only time dependent but also state dependent. Indeed, as shown by [King and Wadhwani (1990), Ramchand and Susmel (1998)], the correlations increase in periods of large volatility. Moreover, [Longin and Solnik (2001)] have proved that the correlations across international equity markets are trend dependent.

- In contrast, a second class of explanation is that correlations between two variables conditioned on signed exceedance (one-sided) or on absolute value (volatility) exceedance of one or both variables may deviate significantly from the unconditional correlation [Boyer et al. (1997), Loretan (2000), Loretan and English (2000)]. In other words, with a fixed unconditional correlation $\rho$, the measured correlation conditioned of a given bullish trend, bearish trend, high or low market volatility, may in general differ from $\rho$ and be a function of the specific market phase. According to this explanation, changes of correlation may be only a fallacious appearance that stems from a change of volatility or a change of trend of the market and not from a real change of unconditional correlation.

The existence of the second class of explanation is appealing by its parsimony, as it posits that observed "changes of correlation" may simply result from the way the measure of dependence is performed. Therefore, before invoking additional mechanisms involving genuine changes of unconditional dependence, it is highly desirable to characterize the different possible ways with which higher or lower conditional dependence can occur in models with constant unconditional dependence. In order to make progress, it is necessary to first distinguish between the different measures of dependence between two variables for large 
or extreme events that have been introduced in the literature, because the conclusions that one can draw about the variability of dependence are sensitive to the choice of its measure. These measures include

1. the correlation conditioned on signed exceedance of one or both variables [Boyer et al. (1997), Loretan (2000), Loretan and English (2000), Cizeau et al. (2001), that we call respectively $\rho_{v}^{+}$and $\rho_{u}$, where $u$ and $v$ denote the thresholds above which the exceedances are calculated,

2. the correlation conditioned on absolute value exceedance (or large volatility), above the threshold $v$, of one or both variables [Boyer et al. (1997), Loretan (2000), Loretan and English (2000), Cizeau et al. (2001)], that we call $\rho_{v}^{s}$ (for a condition of exceedance on one variable),

3. the tail-dependence parameter $\lambda$, which has a simple analytical expression when using copulas [Embrechts et al. (2001), Lindskog (1999)] such as the Gumbel copula [Longin and Solnik (2001]],

4. the spectral measure associated with the tail index (assumed to be the same of all assets) of extreme value multivariate distributions [Davis et al. (1999), Starica (1999), Hauksson et al. (2001)],

5. tail indices of extremal correlations defined as the upper or lower correlation of exceedances of ordered log-values [Quintos (2001)],

6. confidence weighted forecast correlations [Bhansali and Wise (2001)] or algorithmic complexity measures [Mansilla (2001)].

Our contribution to the literature is to provide explicit analytical expressions for several measures (the conditional correlation coefficients $\rho_{v}^{+}, \rho_{v}^{s}, \rho_{u}$, the conditional Spearman's rho $\rho_{s}(v)$ and the tail dependence coefficients $\lambda$ and $\bar{\lambda}$ ) of dependence between two variables for large or extreme events for several models of interest for financial time series. These models are the bivariate Gaussian distribution, the bivariate Student's distribution, and the one factor model for various distributions of the factor. Initially, we hoped to show the existence of logical links between some of these measures, such as a vanishing taildependence parameter $\lambda$ implies vanishing asymptotic $\rho_{v \rightarrow+\infty}^{+}$and $\rho_{u \rightarrow+\infty}$ or $\rho_{s}(v \rightarrow+\infty)$. We show that this turns out to be wrong and one can construct simple examples for which all possible combinations such as for instance $\left(\lambda=0\right.$ and $\left.\rho_{v \rightarrow+\infty}^{+}=0\right),\left(\lambda=0\right.$ and $\left.\rho_{v \rightarrow+\infty}^{+} \neq 0\right),\left(\lambda \neq 0\right.$ and $\left.\rho_{v \rightarrow+\infty}^{+}=0\right)$ and $\left(\lambda \neq 0\right.$ and $\left.\rho_{v \rightarrow+\infty}^{+} \neq 0\right)$ occur. Therefore, each of these measures probe a different quality of the dependence between two variables for large or extreme events. In addition, even if $\rho_{v \rightarrow+\infty}^{+}=0$ and $\rho_{u \rightarrow+\infty}$ are zero, they decay in general extremely slowly as inverse powers of $v$ and $u$ and may thus remain significant for most pratical applications. Somewhat similarly to risks whose adequate characterization requires an extension beyond the restricted one-dimensional measure in terms of the variance (volatility) to include all higher order cumulants or more generally the knowledge of the full distribution [Sornette et al. (2000a), Sornette et al. (2000b), Andersen and Sornette (2001)], our results suggest that taildependence has also a multidimensional character.

One of our goal is also to provide a link between two possible descriptions of financial data. The first one, based on a purely statistical approach, is the multivariate or copula analysis, while the second one, which relies on a principal components analysis, is built on the factor models. As we shall see, the dependence between two variables for large or extreme events may be quite different in these different models with the same margins.

Section 1 describes three conditional correlation coefficients, namely the correlation $\rho_{v}^{+}$conditioned on signed exceedance of one variable, or on both variables $\left(\rho_{u}\right)$ and the correlation $\rho_{v}^{s}$ conditioned on absolute value exceedance (or large volatility) of one variable. [Boyer et al. (1997)] have already provided the general expression of $\rho_{v}^{+}$and $\rho_{v}^{s}$ for the Gaussian bivariate model. We use this result to give their $v$ dependence for large $v: \rho_{v}^{+} \propto 1 / v$ and $1-\rho_{v}^{s} \propto 1 / v^{2}$. We also give an intuitive explanation of these results. We then 
provide the general expression of $\rho_{v}^{+}$and $\rho_{v}^{s}$ for the Student's bivariate model with $\nu$ degrees of freedom. We show that both $\rho_{v}^{+}$and $\rho_{v}^{s}$ go to a constant for large $v$. For the factor model $X=\alpha Y+\epsilon$, we provide a general expression of the conditional correlation coefficient whatever the distributions of $Y$ and $\epsilon$ may be. In the special case when $Y$ has a Student's distribution, we find $1-\rho_{v}^{+} \propto 1-\rho_{v}^{s} \propto 1 / v^{2}$. Conditioning on both variables, we are able to provide the asymptotic dependence of $\rho_{u}$ only for the bivariate Gaussian model: $\rho_{u} \propto 1 / u^{2}$ for $u \rightarrow+\infty$.

In section 2, to account for the deficiencies of the correlation coefficient, we propose an alternative measure of dependence, the conditional Spearman's rho, which is related to the probability of concordance and discordance of several events drawn from the same probability distribution. This measure provides an important improvement with respect to the correlation coefficient since it only takes into account the dependence structure of the variable and is not sensitive to the marginal behavior of each variable. We perform numerical computations to derive the behavior of the conditional Spearman's rho, denoted by $\rho_{s}(v)$. This allows us to prove that there is no direct relation between the Spearman's rho conditioned on large values and the correlation coefficient conditioned on the same values. Therefore, each of these coefficients quantifies a different kind of extreme dependence.

Section 3 discusses the tail-dependence parameters $\lambda$ and $\bar{\lambda}$. We first recall their definitions and values for Gaussian and Student's bivariate distributions of $X$ and $Y$, already known in the literature. For the Gaussian factor model, it is trivial to show that $\lambda=0$. A non-trivial result is obtained for the Student's factor model $X=\alpha Y+\epsilon$ where $Y$ and $\epsilon$ mutually independent: $\lambda$ is found non-zero and a function only of $\alpha$ and of the the scale factor of $\epsilon$. More generally, a theorem established in [Malevergne and Sornette (2002)] allows one to calculate the coefficient of tail dependence for any distribution of the factor and shows that $\lambda$ vanishes for any rapidly varying factor.

Section 4 provides a synthesis and comparison between these different results. A first important message is that there is no unique measure of extreme dependence. Each of the coefficients of extreme dependence that we have studied provides a specific quantification that is sensitive to a certain combination of the marginals and of the copula of the two random variables. Similarly to risks whose adequate characterization requires an extension beyond the restricted one-dimensional measure in terms of the variance (volatility) to include the knowledge of the full distribution, tail-dependence has also a multidimensional character. A second important message is that the increase of some of the coefficients of tail dependence as one goes more in the tails does not necessarily signals a genuine increase of the unconditional correlation or dependence between the two variables. Our calculations firmly confirm that this increase is a general and unvoidable result of the statistical properties of many multivariate models of dependence.

\section{Conditional correlation coefficient}

In this section we discuss the properties of the correlation coefficient conditioned on one variable. We study the difference when conditioning on the signed values or on absolute values $\square$ of the variable. This allows us to conclude that conditioning on signed values is generally more efficient than conditioning on absolute values, and that, as already underlined by [Boyer et al. (1997), for instance], the conditional correlation coefficient suffers from a bias which forbides its use as a tool to measure a change in the correlation between two assets when the volatility increases, as seen in many papers about contagion.

We then present some empirical illustrations of the evolution of the correlation between an asset and the market portfolio. We conclude this section with some considerations about the correlation coefficient conditioned on both variables which allows us to assert that this kind of conditioning does not provide a real

\footnotetext{
${ }^{1}$ Conditioning on absolute value of the variable of interest is only meaningful when its distribution is symetric.
} 
improvement with respect to the correlation coefficient conditioned on one signed variable.

\subsection{Definition}

We study the correlation coefficient $\rho_{\mathcal{A}}$ of two real random variables $X$ and $Y$ conditioned on $Y \in \mathcal{A}$, where $\mathcal{A}$ is a subset of $\mathbb{R}$ such that $\operatorname{Pr}\{Y \in \mathcal{A}\}>0$.

By definition, the conditional correlation coefficient $\rho_{\mathcal{A}}$ is given by

$$
\rho_{\mathcal{A}}=\frac{\operatorname{Cov}(X, Y \mid Y \in \mathcal{A})}{\sqrt{\operatorname{Var}(X \mid Y \in \mathcal{A}) \cdot \operatorname{Var}(Y \mid Y \in \mathcal{A})}} .
$$

Applying this general expression of the conditional correlation coefficient, we will give closed formulae for several standard distributions and models.

\section{$1.2 \quad X$ and $Y$ have a Gaussian distribution}

Let the variables $X$ and $Y$ have a multivariate Gaussian distribution with (unconditional) correlation coefficient $\rho$. The following result have been proved by [Boyer et al. (1997)] :

$$
\rho_{\mathcal{A}}=\frac{\rho}{\sqrt{\rho^{2}+\left(1-\rho^{2}\right) \frac{\operatorname{Var}(Y)}{\operatorname{Var}(Y \mid Y \in \mathcal{A})}}} .
$$

We can note that $\rho$ and $\rho_{\mathcal{A}}$ have the same sign, that $\rho_{\mathcal{A}}=0$ if and only if $\rho=0$ and that $\rho_{\mathcal{A}}$ does not depend directly on $\operatorname{Var}(X)$. Note also that $\rho_{\mathcal{A}}$ can be either greater or smaller than $\rho$ since $\operatorname{Var}(Y \mid Y \in \mathcal{A})$ can be either greater or smaller than $\operatorname{Var}(Y)$. We will illustrate this property in the two following examples.

\subsubsection{Conditioning on $Y>v$}

Let the conditioning set be $\mathcal{A}=[v,+\infty)$, with $v \in \mathbb{R}_{+}$. Thus $\rho_{\mathcal{A}}$ is the correlation coefficient conditioned on $Y$ larger than $v$. It will be denoted by $\rho_{v}^{+}$in the sequel. Assuming for simplicity, but without loss of generality, that $\operatorname{Var}(Y)=1$, we have

$$
\rho_{v}^{+}=\frac{\rho}{\sqrt{\rho^{2}+\frac{1-\rho^{2}}{\operatorname{Var}(Y \mid Y>v)}}} .
$$

Appendix A.1 presents an exact calculation of $\operatorname{Var}(Y \mid Y \in \mathcal{A})$, which leads, for large $v$, to

$$
\rho_{v}^{+} \sim_{v \rightarrow \infty} \frac{\rho}{\sqrt{1-\rho^{2}}} \cdot \frac{1}{v} .
$$

Thus, $\rho_{v}^{+}$goes slowly to zero as $v$ goes to infinity. Obviously, by symmetry, the conditional correlation coefficient $\rho_{v}^{-}$, conditioned on $Y$ smaller than $v$, obeys the same formula. 


\subsubsection{Conditioning on $|Y|>v$}

Let now the conditioning set be $\mathcal{A}=(-\infty,-v] \cup[v,+\infty)$, with $v \in \mathbb{R}_{+}$. Thus $\rho_{\mathcal{A}}$ is the correlation coefficient conditioned on $|Y|$ larger than $v$, i.e., it is conditioned on large volatility of $Y$. Still assuming $\operatorname{Var}(Y)=1$, we denote it by $\rho_{v}^{s}$ :

$$
\rho_{v}^{s}=\frac{\rho}{\sqrt{\rho^{2}+\frac{1-\rho^{2}}{\operatorname{Var}(Y|| Y \mid>v)}}} .
$$

Appendix A.2 presents the calculation of $\operatorname{Var}(Y|| Y \mid>V)$, which allows us to conclude that, for large $v$,

$$
\rho_{v}^{s} \sim_{v \rightarrow \infty} \frac{\rho}{\sqrt{\rho^{2}+\frac{1-\rho^{2}}{2+v^{2}}}},
$$

showing that $\rho_{v}^{s}$ goes to one as $v$ goes to infinity as $1-\rho_{v}^{s} \sim_{v \rightarrow \infty} \frac{1-\rho^{2}}{\rho^{2}} v^{-2}$.

\subsubsection{Conditioning on $Y>v$ versus $|Y|>v$}

In the case of two Gaussian random variables, the two conditional correlation coefficients $\rho_{v}^{+}$and $\rho_{v}^{s}$ thus exhibit opposite behavior since the conditional correlation coefficient $\rho_{v}^{+}$is a decreasing function of $v$ which goes to zero as $v \rightarrow+\infty$ while the conditional correlation coefficient $\rho_{v}^{s}$ is an increasing function of $v$ and goes to one as $v \rightarrow \infty$.

Let us provide an intuitive explanation (see also [Longin and Solnik (1995)]). As seen from (3), $\rho_{v}^{+}$is controlled by the dependence $\operatorname{Var}(Y \mid Y>v) \propto 1 / v^{2}$ derived in Appendix A.1. In contrast, as seen from (5), $\rho_{v}^{s}$ is controlled by $\operatorname{Var}(Y|| Y \mid>v) \propto v^{2}$ given in Appendix A.2. The difference between $\rho_{v}^{+}$and $\rho_{v}^{s}$ can thus be traced back to that between $\operatorname{Var}(Y \mid Y>v) \propto 1 / v^{2}$ and $\operatorname{Var}(Y|| Y \mid>v) \propto v^{2}$ for large $v$.

This results from the following effect. For $Y>v$, one can picture the possible realizations of $Y$ as those of a random particle on the line, which is strongly attracted to the origin by a spring (the Gaussian distribution that prevents $Y$ from performing significant fluctuations beyond a few standard deviations) while being forced to be on the right to a wall at $Y=v$. It is clear that the fluctuations of the position of this particle are very small as it is strongly glued to the unpenetrable wall by the restoring spring, hence the result $\operatorname{Var}(Y \mid Y>v) \propto 1 / v^{2}$. In constrast, for the condition $|Y|>v$, by the same argument, the fluctuations of the particle are hindered to be very close to $|Y|=v$, i.e., very close to $Y=+v$ or $Y=-v$. Thus, the fluctuations of $Y$ typically flip from $-v$ to $+v$ and vice-versa. It is thus not surprising to find $\operatorname{Var}(Y|| Y \mid>$ $v) \propto v^{2}$.

This argument makes intuitive the results $\operatorname{Var}(Y \mid Y>v) \propto 1 / v^{2}$ and $\operatorname{Var}(Y|| Y \mid>v) \propto v^{2}$ for large $v$ and thus the results for $\rho_{v}^{+}$and for $\rho_{v}^{s}$ if we use (3) and (5). We now attempt to justify $\rho_{v}^{+} \sim_{v \rightarrow \infty} \frac{1}{v}$ and $1-\rho_{v}^{s} \sim_{v \rightarrow \infty} 1 / v^{2}$ directly by the following intuitive argument. Using the picture of particles, $X$ and $Y$ can be visualized as the positions of two particles which fluctuate randomly. Their joint bivariate Gaussian distribution with non-zero unconditional correlation amounts to the existence of a spring that ties them together. Their Gaussian marginals also exert a spring-like force attaching them to the origin. When $Y>v$, the $X$-particle is teared off between two extremes, between 0 and $v$. When the unconditional correlation $\rho$ is less than 1 , the spring attracting to the origin is stronger than the spring attracting to the wall at $v$. The particle $X$ thus undergoes tiny fluctuations around the origin that are relatively less and less attracted by the $Y$-particle, hence the result $\rho_{v}^{+} \sim_{v \rightarrow \infty} \frac{1}{v} \rightarrow 0$. In constrast, for $|Y|>v$, notwithstanding the still strong attraction of the $X$-particle to the origin, it can follow the sign of the $Y$-particle without paying too much cost in matching its amplitude $|v|$. Relatively tiny fluctuation of the $X$-particle but of the same sign as $Y \approx \pm v$ will result in a strong $\rho_{v}^{s}$, thus justifying that $\rho_{v}^{s} \rightarrow 1$ for $v \rightarrow+\infty$. 


\section{3 $X$ and $Y$ have a Student's distribution}

Let the variables $X$ and $Y$ have a multivariate Student's distribution with $\nu$ degrees of freedom and an (unconditional) correlation coefficient $\rho$. According to the proposition stated in appendix B.1, we have

$$
\rho_{\mathcal{A}}=\frac{\rho}{\sqrt{\rho^{2}+\frac{\mathrm{E}\left[\mathrm{E}\left(X^{2} \mid Y\right)-\rho^{2} Y^{2} \mid Y \in \mathcal{A}\right]}{\operatorname{Var}(Y \mid Y \in \mathcal{A})}}} .
$$

Appendix B.1 gives the explicit formulas of $\mathrm{E}\left[\mathrm{E}\left(X^{2} \mid Y\right)-\rho^{2} Y^{2} \mid Y \in \mathcal{A}\right]$ and $\operatorname{Var}(Y \mid Y \in \mathcal{A})$.

Expression (7) is the analog for a Student bivariate distribution to (2) derived above for the Gaussian bivariate distribution. Again, $\rho$ and $\rho_{\mathcal{A}}$ share the following properties: they have the same sign, $\rho_{\mathcal{A}}$ equals zero if and only if $\rho$ equals zero and $\rho_{\mathcal{A}}$ can be either greater or smaller than $\rho$. We now apply this general formula $(\nabla)$ to the calculus of $\rho_{v}^{+}$and $\rho_{v}^{s}$.

\subsubsection{Conditioning on $Y>v$}

We consider the conditioning set $\mathcal{A}=[v,+\infty)$ with $v \in \mathbb{R}_{+}$. The expressions of $\mathrm{E}\left[\mathrm{E}\left(X^{2} \mid Y\right)-\rho^{2} Y^{2} \mid Y \in\right.$ $\mathcal{A}]$ and $\operatorname{Var}(Y \mid Y \in \mathcal{A})$, in such a case, can be obtained in closed form and are given in appendix B.3. This allow us to conclude that, for large $v$,

$$
\rho_{v}^{+} \longrightarrow \frac{\rho}{\sqrt{\rho^{2}+(\nu-1) \sqrt{\frac{\nu-2}{\nu}}\left(1-\rho^{2}\right)}},
$$

which is a non vanishing contant, excepted for $\rho=0$. Moreover, for $\nu$ larger than $\nu_{c} \simeq 2.839$, this constant is smaller than the unconditional correlation coefficient $\rho$, for all value of $\rho$.

\subsubsection{Conditioning on $|Y|>v$}

The conditioning set is now $\mathcal{A}=(-\infty,-v] \cup[v,+\infty)$, with $v \in \mathbb{R}_{+}$. Appendix B.4 gives the closed expressions of $\mathrm{E}\left[\mathrm{E}\left(X^{2} \mid Y\right)-\rho^{2} Y^{2} \mid Y \in \mathcal{A}\right]$ and $\operatorname{Var}(Y \mid Y \in \mathcal{A})$. For large $v$, this leads to

$$
\rho_{v}^{s} \longrightarrow \frac{\rho}{\sqrt{\rho^{2}+\frac{1}{(\nu-1)} \sqrt{\frac{\nu-2}{\nu}}\left(1-\rho^{2}\right)}}
$$

which is again a non vanishing contant. Contrarily to the previous case, this constant is alway larger than $\rho$, whatever $\nu$ (larger than two) may be.

\subsubsection{Conditioning on $Y>v$ versus on $|Y|>v$}

The results (8) and (9) are valid for $\nu>2$, as one can expect since the second moment has to exist for the correlation coefficient to be defined. We remark that here, contrarily to the Gaussian case, the conditioning set is not really important. Indeed with both conditioning set, $\rho_{v}^{+}$and $\rho_{v}^{s}$ goes to a constant different from zero and one, when $v$ goes to infinity. This striking difference can be explained by the large fluctuations allowed by the Student's distribution, and can be related to the fact that the coefficient of tail dependence for this distribution does not vanish even though the variables are anti-correlated (see section 3.2 below). 
Contrarily to the Gaussian distribution which binds the fluctuations of the variables near the origin, the Student's distribution allows for 'wild' fluctuations. These properties are thus responsible for the result that, contrarily to the Gaussian case for which the conditional correlation coefficient goes to zero when conditioned on large signed values and goes to one when conditioned on large unsigned values, the conditional correlation coefficient for Student's variables have a similar behavior in both cases. Intuitively, the large fluctuations of $X$ for large $v$ dominate and control the asymptotic dependence.

\section{4 $X$ and $Y$ are related by a linear equation (factor model)}

We now assume that $X$ and $Y$ are two random variables following the equation

$$
X=\alpha Y+\epsilon,
$$

where $\alpha$ is a non random real coefficient and $\epsilon$ an idiosyncratic noise independent of $Y$, whose distribution admits a centered moment of second order $\sigma_{\epsilon}^{2}$. Let us also denote by $\sigma_{y}^{2}$ the second centered moment of the variable $Y$. This kind of relation between $X$ and $Y$ is the so-called one factor model whose applications in finance can be traced back to [Ross (1976)]. This one factor model with independence between $Y$ and $\epsilon$ is of course naive for concrete applications, as it neglects the potential influence of other factors in the determination of $X$. But for our purpose, it provides a simple illustrative model with rich and somewhat surprising results.

Appendix $\mathrm{G}$ shows that the conditional correlation coefficient of $X$ and $Y$ is

$$
\rho_{\mathcal{A}}=\frac{\rho}{\sqrt{\rho^{2}+\left(1-\rho^{2}\right) \frac{\operatorname{Var}(y)}{\operatorname{Var}(y \mid y \in \mathcal{A})}}},
$$

where

$$
\rho=\frac{\alpha \cdot \sigma_{y}}{\sqrt{\alpha^{2} \cdot \sigma_{y}^{2}+\sigma_{\epsilon}^{2}}}
$$

denotes the unconditional correlation coefficient of $X$ and $Y$. Note that the term $\sigma_{\epsilon}^{2}$ in the expression (12) of $\rho$ is the only place where the influence of the idiosynchratic noise is felt.

Expression (11) is the same as (2) for the bivariate Gaussian situation studied in 1.2. This is not surprising since, in the case where $Y$ and $\epsilon$ have univariate Gaussian distributions, the joint distribution of $X$ and $Y$ is a bivariate Gaussian distribution. The new fact is that this expression (11) remains true whatever the distribution of $Y$ and $\epsilon$, provided that their second moments exist.

We now present the asymptotic expression of $\rho_{\mathcal{A}}$ for $Y$ with a Gaussian or a Student's distribution. Note that the expression of $\rho_{\mathcal{A}}$ is simple enough to allow for exact calculations for a larger class of distributions.

\subsection{1 $Y$ has a Gaussian distribution}

Let us assume that $Y$ has a Gaussian distribution, while the distribution of $\epsilon$ can be everything (provided that $\left.\mathrm{E}\left[\epsilon^{2}\right]<\infty\right)$. The variance $\operatorname{Var}\left(Y \mid Y>v\right.$ ) behaves like $1 / v^{2}$ for $v$ large enough according to (41) while $\operatorname{Var}(Y|| Y \mid>v) \sim v^{2}$ according to (42). This obviously yields the same results as these given by equations (4) and (6). 


\subsection{2 $Y$ has a Student's distribution}

Let us assume that $Y$ has a Student's distribution. Both $\operatorname{Var}(Y \mid Y>v)$ and $\operatorname{Var}(Y|| Y \mid>v)$ are proportional to $v^{2}$ for $v$ large enough, according to (107) and (111). This leads to

$$
\rho_{v}^{+, s} \sim \frac{1}{\sqrt{1+\frac{K}{v^{2}}}},
$$

where $K$ is a positive constant. $\rho_{v}^{+, s}$ thus goes to 1 as $v$ goes to infinity with $1-\rho_{v}^{+, s} \propto 1 / v^{2}$.

\subsection{Empirical evidence}

Figures 1 and 2 show the conditional correlation coefficient between the daily returns for the Disney Company and the Standard \& Poor's 500 Index (fig 11) and between the daily returns of the Pepsico Incorporated and the Standard \& Poor's 500 Index (fig 2). For both figures, the upper panel depicts the correlation coefficient conditional on the daily returns of the Standard \& Poor's 500 Index larger than (resp. smaller than) a given positive (resp. negative) value $v$, while the lower panel gives the same information when the conditioning is on the stock returns. The two thin curves in each panel gives the values beyond which the estimated correlation coefficient is significantly different from zero, at the $95 \%$ confidence level.

We observe that Pepsico and the Standard \& Poor's 500 Index shows no significant correlation, neither in the upper nor in the lower tail, whatever the conditioning variable may be. In contrast, conditioned on large fluctuations of the Standard \& Poor's, the correlation coefficient between Disney and the Standard \& Poor's remains very significant. When conditioning on large negative fluctuations of Disney, this correlation remains strong. However, for large positive returns of Disney, the conditional correlation sinks within the statistical uncertainty. This last feature is found more generally for essentially all pairs of stocks and indices that we have explored, for which the conditional correlation is weak or non-existent for large positive returns. There is thus a strong and significant asymmetry between the correlations conditioned on negative compared to positive moves.

We stress that it would be incorrect to conclude that the correlation increases between Disney and the Standard \& Poor's, based solely on the empirical determination of the correlation coefficient conditioned on large returns of the Standard \& Poor's. Indeed, we have shown by explicit analytical calculations that the conditional correlations can increase when conditioning on larger fluctuations without needing any variation of the unconditional correlation coefficient.

Thus, the conditional correlations do not appear to be very useful tools for probing the possible changes in the dependence structure between two assets. However, coupled with the Capital Asset Pricing Model [Sharpe (1964)], it provides interesting insights. The CAPM predicts that the return of any asset $X$ can be explained by the market return $Y$ according to the relation

$$
X=\beta \cdot Y+\epsilon,
$$

where $\beta$ is a constant and $\epsilon$ is an idiosyncratic noise. As an illustration, we will study the extreme dependence generated by this model and compare it with the true observed dependence. Figure 3 (respectively figure ఐ) shows in thick line the correlation coefficient between Disney (respectively Pepsico) and the Standard \& Poor's, both conditioned on the Standard \& Poor's fluctuations. We have also performed the bootstrap estimation of the $95 \%$ confidence interval, represented for the two thin lines in figures 3 and 4 , of the conditional correlation coefficent predicted by the factor model (14). The CAPM is found to provide a very good description of the extreme dependence between Disney and the market, while it completely fails to 
account for the lack of extreme dependence between Pepsico and the market. The failure of the factor model to account for the conditional correlation between Pepsico and the Standard \& Poor's index may be due to the impact of additional factors that have been omitted in this one-factor model.

It is important to stress that the observed increase in conditional correlation between Disney and the Standard \& Poor's is not due to a genuine increase in the unconditional correlation but results only from the interplay between the conditioning and the dependence structure of the factor model.

In summary, the conditional correlation coefficient is an unreliable tool, to say the least, for characterizing possible changes in the dependence structure when the volatility level increases, as it is often read in the contagion literature.

\subsection{Conditional correlation coefficient on both variables}

\subsubsection{Definition}

We consider two random variables $X$ and $Y$ and define their conditional correlation coefficient $\rho_{\mathcal{A}, \mathcal{B}}$, conditioned upon $X \in \mathcal{A}$ and $Y \in \mathcal{B}$, where $\mathcal{A}$ and $\mathcal{B}$ are two subsets of $\mathbb{R}$ such that $\operatorname{Pr}\{X \in \mathcal{A}, Y \in \mathcal{B}\}>0$, by

$$
\rho_{\mathcal{A}, \mathcal{B}}=\frac{\operatorname{Cov}(X, Y \mid X \in \mathcal{A}, Y \in \mathcal{B})}{\sqrt{\operatorname{Var}(X \mid X \in \mathcal{A}, Y \in \mathcal{B}) \cdot \operatorname{Var}(Y \mid X \in \mathcal{A}, Y \in \mathcal{B})}} .
$$

In this case, it is much more difficult to obtain general results for any specified class of distributions compared to the previous case of conditioning on a single variable. We have only been able to give the asymptotic behavior for a Gaussian distribution in the situation detailed below, using the expressions in [Oohnson and Kotz (1972), p 113] or proposition A.1 of [Ang and Chen (2001)].

\subsubsection{Conditioning on $X$ and $Y$ larger than $u$ for bivariate Gaussian random variables}

Let us assume that the pair of random variables $(\mathrm{X}, \mathrm{Y})$ has a Normal distribution with unit unconditional variance and unconditional correlation coefficient $\rho$. The subsets $\mathcal{A}$ and $\mathcal{B}$ are both choosen equal to $[u,+\infty)$, with $u \in \mathbb{R}_{+}$. Let us denote by $\rho_{u}$ the correlation coefficient conditional on this particular choice for the subsets $\mathcal{A}$ and $\mathcal{B}$ :

$$
\rho_{u}=\frac{\operatorname{Cov}\left(X, Y \mid(X, Y) \in[u,+\infty)^{2}\right)}{\sqrt{\operatorname{Var}\left(X \mid(X, Y) \in[u,+\infty)^{2}\right) \cdot \operatorname{Var}\left(Y \mid(X, Y) \in[u,+\infty)^{2}\right)}} .
$$

Under the assumptions stated above, Appendix A.3 shows that, for large $u$ :

$$
\rho_{u} \sim_{u \rightarrow \infty} \rho \frac{1+\rho}{1-\rho} \cdot \frac{1}{u^{2}}
$$

which goes to zero. This decay is faster than in the case governed by (4) resulting from the conditioning on a single variable leading to $\rho_{v}^{+} \sim_{v \rightarrow+\infty} 1 / v$, but, as we expect, we do not observe a significant change. Thus, the correlation coefficient conditioned on both variables does not yield new information and does not bring any special improvement with respect to the correlation coefficient conditioned on a single variable. 


\section{Conditional concordance measures}

The (conditional) correlation coefficient, which has just been investigated, suffers from several deficiencies. First, it is only a measure of linear dependence. Thus, as stressed by [Embrechts et al. (1999)], it is fully satisfying only for the description of the dependence of variables with elliptical distributions. Moreover, the correlation coefficient aggregates the information contained both in the marginal and in the collective behavior. The correlation coefficient is not invariant under an increasing change of variable, a transformation which is known to let unchanged the dependence structure. Thus, it is desirable to find another measure of the dependence between two assets or more generally between two random variables, which, contrarily to the linear correlation coefficient, only depends on the copula properties, and are therefore not affected by a change in the marginal distributions (provided that the mapping is increasing). It turns out that this desirable property is shared by all concordance measures. Among these measures are the well-known Kendall's tau, Spearman's rho or Gini's beta (see [Nelsen (1998)] for details).

However, these concordance measures are not well-adapted, as such, to the study of extreme dependence, because they are functions of the whole distribution, including the moderate and small returns. A simple idea to investigate the extreme concordance properties of two random variables is to calculate these quantities conditioned on values larger than a given threshold and let this threshold go to infinity.

In the sequel, we will only focus on the Spearman's rho which can be easily estimated empirically. It offers a natural generalization of the (linear) correlation coefficient. Indeed, the correlation coefficient quantifies the degres of linear dependence between two random variables, while the Spearman's rho quantifies the degree of functional dependence, whatever the functional dependence between the two random variables may be. This represents a very interesting improvement. Perfect correlations (resp. anti-correlation) give a value 1 (resp. -1) both for the standard correlation coefficient and for the Spearman's rho. Otherwise, there is no general relation allowing to deduce the Spearman's rho from the correlation coefficient and vice-versa.

\subsection{Definition}

The Spearman's rho, denoted by $\rho_{s}$ in the sequel, measures the difference between the probability of concordance and the probability of discordance for the two pairs of random variables $\left(X_{1}, Y_{1}\right)$ and $\left(X_{2}, Y_{3}\right)$, where the pairs $\left(X_{1}, Y_{1}\right),\left(X_{2}, Y_{2}\right)$ and $\left(X_{3}, Y_{3}\right)$ are three independent realizations drawn from the same distribution:

$$
\rho_{s}=3\left(\operatorname{Pr}\left[\left(X_{1}-X_{2}\right)\left(Y_{1}-Y_{3}\right)>0\right]-\operatorname{Pr}\left[\left(X_{1}-X_{2}\right)\left(Y_{1}-Y_{3}\right)<0\right]\right) .
$$

The Spearman's rho can also be expressed with the copula $C$ of the two variables X and Y (see [Nelsen (1998)], for instance):

$$
\rho_{s}=12 \int_{0}^{1} \int_{0}^{1} C(u, v) d u d v-3
$$

which allows us to easily calculate $\rho_{s}$ when the copula $C$ is known in closed form.

Denoting $U=F_{X}(X)$ and $V=F_{Y}(V)$, it is easy to show that $\rho_{s}$ is nothing but the (linear) correlation coefficient of the uniform random variables $U$ and $V$ :

$$
\rho_{s}=\frac{\operatorname{Cov}(U, V)}{\sqrt{\operatorname{Var}(U) \operatorname{Var}(V)}} .
$$

This justifies its name as a correlation coefficient of the rank, and shows that it can easily be estimated. 
An attractive feature of the Spearman's rho is to be independent of the margins, as we can see in equation (19). Thus, contrarily to the linear correlation coefficient, which aggregates the marginal properties of the variables with their collective behavior, the rank correlation coefficient takes into account only the dependence structure of the variables.

Using expression (20), we propose a natural definition of the conditional rank correlation, conditioned on $V$ larger than a given threshold $\tilde{v}$ :

$$
\rho_{s}(\tilde{v})=\frac{\operatorname{Cov}(U, V \mid V \geq \tilde{v})}{\sqrt{\operatorname{Var}(U \mid V \geq \tilde{v}) \operatorname{Var}(V \mid \geq \tilde{v})}} .
$$

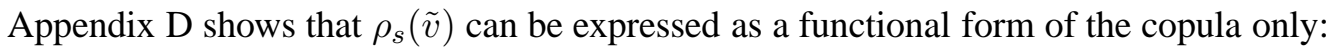

$$
\rho_{s}(\tilde{v})=\frac{\frac{12}{1-\tilde{v}} \int_{\tilde{v}}^{1} d v \int_{0}^{1} d u C(u, v)-6 \int_{0}^{1} d u C(u, \tilde{v})-3}{\sqrt{1-4 \tilde{v}+24(1-\tilde{v}) \int_{0}^{1} d u u C(u, \tilde{v})+12(2 \tilde{v}-1) \int_{0}^{1} d u C(u, \tilde{v})-12\left(\int_{0}^{1} d u C(u, \tilde{v})\right)^{2}}} .
$$

\subsection{Example}

Contrarily to the conditional correlation coefficient, we have not been able to obtain analytical expressions for the conditional Spearman's rho, at least for the distributions that we have considered up to now. Obviously, for many families of copulas known in closed form, equation (22) allows for an explicit calculation of $\rho_{s}(v)$. However, most copulas of interest in finance have no simple closed form, so that it is necessary to resort to numerical computations.

As an example, let us consider the bivariate Gaussian distribution (or copula) with unconditional correlation coefficent $\rho$. It is well-known that its unconditional Spearman's rho is given by

$$
\rho_{s}=\frac{6}{\pi} \cdot \arcsin \frac{\rho}{2} .
$$

Figure 5 shows the conditional Spearman's rho $\rho_{s}(v)$ defined by (21) obtained from a numerical integration of (22). We observe the same bias as for the conditional correlation coefficient, namely the conditional rank correlation changes with $v$ eventhough the unconditional correlation is fixed to a constant value. Nonetheless, this conditional Spearman's rho seems more sensitive than the conditional correlation coefficient since we can observe in figure 5 that, as $v$ goes to one, the conditional Spearman's rho $\rho_{s}(v)$ does not go to zero for all values of $\rho$, as previously observed with the conditional correlation coefficient (see equation (4)).

\subsection{Empirical evidence}

In figures 6 and 7 we have represented the conditionnal Spearman's rho for the same assets as in figures 1 and 2. The results are strikingly different compared to figures (1) and (2). While the conditional correlation coefficient would lead to conclude positively on the existence of significant extreme correlations between Disney and the Standard \& Poor's 500 index, the conditional Spearman's rho leads to the opposite conclusion. Specifically, figure 1 suggests that the correlation coefficient conditioned on large negative Standard $\&$ Poor's returns converges to a value very close to 1 . Since Spearman's rho (conditioned or not) goes to 1 when the correlation coefficient (conditioned or not) goes to 1, this predicts that the Spearman's rho conditioned on large negative Standard \& Poor's returns should also converge to 1. We observe the opposite behavior that the Spearman's rho is not significantly different from zero in the lowest quantiles. 
This contradictory empirical result puts in light the problem of obtaining reliable and sensitive estimations of such correlation measures. In particular, the Pearson's coefficient usually employed to estimate the correlation coefficient between two variables is known to be not very efficient when the variables are fat-tailed and when the estimation performed on a small sample. Indeed, with small samples, the Pearson's coefficient is very sensitive to the largest value, which can lead to an important bias in the estimation.

Moreover, even with large sample size, Meerschaert and Scheffler (2001)] have shown that the nature of the convergence between the Pearson's coefficient of two times series with tail index $\mu$ and the theoretical correlation as the sample size $T$ tends to infinity is sensitive to the existence and strength of the theoretical correlation. If there is no theoretical correlation between the two times series, the sample correlation tends to zero with Gaussian fluctuations. If the theoretical correlation is non-zero, the difference between the sample correlation and the theoretical correlation times $T^{1-2 / \mu}$ converges in distribution to a stable law with index $\mu / 2$.

\section{Tail dependence}

For the sake of completeness, and since it is directly related to the multivariate extreme values theory, we study the so-called coefficient of tail dependence $\lambda$. To our knowledge, its interest for financial applications has been first underlined by [Embrechts et al. (2001)].

The coefficient of tail dependence characterizes an important property of the extreme dependence between $X$ and $Y$, using the (original or unconditional) copula of $X$ and $Y$. In constrast, the conditional spearman's rho is defined in terms of a conditional copula, as it can be seen as the "unconditional Spearman's rho" of the copula of $X$ and $Y$ conditioned on $Y$ larger than the threshold $v$. This copula of $X$ and $Y$ conditioned on $Y$ larger than the threshold $v$ is not the true copula of $X$ and $Y$ because it is modified by the conditioning. In this sense, the tail dependence parameter $\lambda$ is a more natural property directly related to the copula of $X$ and $Y$.

To begin with, we recall the definition of the coefficient $\lambda$ as well as of $\bar{\lambda}$ (see below) which allows one to quantify the amount of dependence in the tail. Then, we present several results concerning the coefficient $\lambda$ of tail dependence for various distributions and models, and finally, we discuss the problems encountered in the estimation of these quantities.

\subsection{Definition}

The concept of tail dependence is appealing by its simplicity. By definition, the (upper) tail dependence coefficient is:

$$
\lambda=\lim _{u \rightarrow 1} \operatorname{Pr}\left[X>F_{X}^{-1}(u) \mid Y>F_{Y}^{-1}(u)\right],
$$

and quantifies the probability to observe a large $X$, assuming that $Y$ is large itself. For a survey of the properties of the tail dependence coefficient, the reader is refered to [Coles et al. (1999), Embrechts et al. (2001), Lindskog (1999), for instance. In words, given that $Y$ is very large (which occurs with probability $1-u$ ), the probability that $X$ is very large at the same probability level $u$ defines asymptotically the tail dependence coefficient $\lambda$.

One of the appeal of this definition of tail dependence is that it is a pure copula property, i.e., it is independent of the margins of $X$ and $Y$. Indeed, let $C$ be the copula of the variables $X$ and $Y$, then if the bivariate copula $C$ is such that

$$
\lim _{u \rightarrow 1} \frac{1-2 u+C(u, u)}{1-u}=\lim _{u \rightarrow 1} 2-\frac{\log C(u, u)}{\log u}=\lambda
$$


exists, then $C$ has an upper tail dependence coefficient $\lambda$ (see [Coles et al. (1999), Embrechts et al. (2001), Lindskog (1999)]).

If $\lambda>0$, the copula presents tail dependence and large events tend to occur simultaneously, with the probability $\lambda$. On the contrary, when $\lambda=0$, the copula has no tail dependence, in this sense, and the variables $X$ and $Y$ are said asymptotically independent. There is however a subtlety in this definition (24) of tail dependence. To make it clear, first consider the case where for large $X$ and $Y$ the cumulative distribution function $H(x, y)$ factorizes such that

$$
\lim _{x, y \rightarrow \infty} \frac{F(x, y)}{F_{X}(x) F_{Y}(y)}=1
$$

where $F_{X}(x)$ and $F_{Y}(y)$ are the margins of $X$ and $Y$ respectively. This means that, for $X$ and $Y$ sufficiently large, these two variables can be considered as independent. It is then easy to show that

$$
\begin{aligned}
\lim _{u \rightarrow 1} \operatorname{Pr}\left\{X>F_{X}^{-1}(u) \mid Y>F_{Y}^{-1}(u)\right\} & =\lim _{u \rightarrow 1} 1-F_{X}\left(F_{X}^{-1}(u)\right) \\
& =\lim _{u \rightarrow 1} 1-u=0
\end{aligned}
$$

so that independent variables really have no tail dependence $\lambda=0$, as one can expect.

However, the result $\lambda=0$ does not imply that the multivariate distribution can be automatically factorized asymptotically, as shown by the Gaussian example. Indeed, the Gaussian multivariate distribution does not have a factorizable multivariate distribution, even asymptotically for extreme values, since the non-diagonal term of the quadratic form in the exponential function does not become negligible in general as $X$ and $Y$ go to infinity. Therefore, in a weaker sense, there may still be a dependence in the tail even when $\lambda=0$.

To make this statement more precise, following [Coles et al. (1999]], let us introduce the coefficient

$$
\begin{aligned}
\bar{\lambda} & =\lim _{u \rightarrow 1} \frac{2 \log \operatorname{Pr}\left\{X>F_{X}^{-1}(u)\right\}}{\log \operatorname{Pr}\left\{X>F_{X}^{-1}(u), Y>F_{Y}^{-1}(u)\right\}}-1 \\
& =\lim _{u \rightarrow 1} \frac{2 \log (1-u)}{\log [1-2 u+C(u, u)]}-1 .
\end{aligned}
$$

It can be shown that the coefficient $\bar{\lambda}=1$ if and only if the coefficient of tail dependence $\lambda>0$, while $\bar{\lambda}$ takes values in $[-1,1)$ when $\lambda=0$, allowing us to quantify the strength of the dependence in the tail in such a case. In fact, it has been established that, when $\bar{\lambda}>0$, the variables $X$ and $Y$ are simultaneously large more frequently than independent variables, while simultaneous large deviations of $X$ and $Y$ occur less frequenlty than under independence when $\bar{\lambda}<0$ (the interested reader is refered to [Ledford and Tawn (1996), Ledford and Tawn (1998]]).

To summarize, independence (factorization of the bivariate distribution) implies no tail dependence $\lambda=0$. But $\lambda=0$ is not sufficient to imply factorization and thus true independence. It also requires, as a necessary condition, that $\bar{\lambda}=0$.

We will first recall the expression of the tail dependence coefficient for usual distributions, and then calculate it in the case of a one-factor model for different distributions of the factor.

\subsection{Tail dependence for Gaussian distributions and Student's distributions}

Assuming that $(X, Y)$ are normally distributed with correlation coefficient $\rho$, Embrechts et al. (2001)] shows that for all $\rho \in[-1,1), \lambda=0$, while [Heffernan (2000] gives $\bar{\lambda}=\rho$, which expresses, as one can expect, that extremes appear more likely together for positively correlated variables. 
In constrast, if $(X, Y)$ have a Student's distribution, [Embrechts et al. (2001)] shows that the tail dependence coefficient is

$$
\lambda=2 \cdot \bar{T}_{\nu+1}\left(\sqrt{\nu+1} \sqrt{\frac{1-\rho}{1+\rho}}\right),
$$

which is greater than zero for all $\rho>-1$, and thus $\bar{\lambda}=1$. This last example prooves that extremes appear more likely together whatever the correlation coefficient may be, showing that, in fact, it does not exist any general relation between the asymptotic dependence and the linear correlation coeffient.

Let us add that these two distributions are elliptical distributions, and that the coefficients of tail dependence obtained in the two previous cases are characteristic of the coefficient of tail dependence observed for this class of distributions. Indeed, [Hult and Lindskog (2001)] have shown that ellipticaly distributed random variables presents tail dependence if and only if they are regularly varing, i.e behaves asymptotically like power laws with exponent $\nu$. In such a case, for every regularly varying pair of random variables elliptically distributed, we have

$$
\lambda=\frac{\int_{\pi / 2-\arcsin \rho}^{\pi / 2} d t \cos ^{\nu} t}{\int_{0}^{\pi / 2} d t \cos ^{\nu} t} .
$$

Note that necessarily, the rhs of equations (31) and (32) are equal, which is natural since the correlation coefficient is an invariant quantity in the class of elliptical distributions and that the coefficient of tail dependence is only determined by the asymptotic behavior of the distribution, so that it does not matter that the distribution is a Student's distribution with $\nu$ degrees of freedom or any other elliptical distribution with the same behavior in the tail.

\subsection{Tail dependence generated by a factor model}

Consider the one-factor model

$$
X=\alpha Y+\epsilon,
$$

where $\epsilon$ is a random variable independent of $Y$ and $\alpha$ a non-random positive coefficient. We now study the tail dependence coefficient $\lambda$ between the two random variables $X$ and $Y$ in two simple cases.

\subsubsection{The Gaussian case}

This case is trivial. Indeed, assume that the factor $Y$ and the idiosyncratic noise $\epsilon$ have Gaussian distributions, it is then obvious that the bivariate distribution of $(X, Y)$ is Gaussian. Thus, the tail dependence coefficient generated by a one factor model, with a Gaussian factor equals zero.

In fact, as we shall see in the sequel, this result remains true for all distributions of the idiosyncratic noise $\epsilon$.

\subsubsection{The Student's case}

Let us assume now that the factor $Y$ and the idiosyncratic noise $\epsilon$ have centered Student's distributions with the same number $\nu$ of degrees of freedom and scale factors respectively equal to 1 and $\sigma$. The choice of the scale factor equal to 1 for $Y$ is not restrictive but only provides a convenient normalization for $\sigma$. Appendix E shows that the tail dependence coefficient is

$$
\lambda=\frac{1}{1+\left(\frac{\sigma}{\alpha}\right)^{\nu}} .
$$


As is reasonable intuitively, the larger the typical scale $\sigma$ of the fluctuation of $\epsilon$ and the weaker is the coupling coefficient $\alpha$, the smaller is the tail dependence.

Let us recall that the unconditional correlation coefficient $\rho$ can be writen as $\rho=\left(1+\frac{\sigma^{2}}{\alpha^{2}}\right)^{-1 / 2}$, which allows us to rewrite the coefficient of upper tail dependence as

$$
\lambda=\frac{\rho^{\nu}}{\rho^{\nu}+\left(1-\rho^{2}\right)^{\nu / 2}} .
$$

Surprinsingly, $\lambda$ does not go to zero for all $\rho$ 's as $\nu$ goes to infinity, as one would expect intuitively. Indeed, a natural reasoning would be that, as $\nu$ goes to infinity, the Student's distribution goes to the Gaussian distribution. Therefore, one could a priori expect to find again the result given in the previous section for the Gaussian factor model. We note that $\lambda \rightarrow 0$ when $\nu \rightarrow \infty$ for all $\rho$ 's smaller than $1 / \sqrt{2}$. But, and here lies the surprise, $\lambda \rightarrow 1$ for all $\rho$ larger than $1 / \sqrt{2}$ when $\nu \rightarrow \infty$. This counter-intuitive result is due to a non-uniform convergence which makes the order to two limits non-commutative: taking first the limit $u \rightarrow 1$ and then $\nu \rightarrow \infty$ is different from taking first the limit $\nu \rightarrow \infty$ and then $u \rightarrow 1$. In a sense, by taking first the limit $u \rightarrow 1$, we always ensure somehow the power law regime even if $\nu$ is later taken to infinity. This is different from first "sitting" on the Gaussian limit $\nu \rightarrow \infty$. It then is a posteriori reasonable that the absence of uniform convergence is made strongly apparent in its consequences when measuring a quantity probing the extreme tails of the distributions.

As an illustration, figure 8 represents the coefficient of tail dependence for the Student's copula and Student's factor model as a function of $\rho$ for various value of $\nu$. It is interesting to note that $\lambda$ equals zero for all negative $\rho$ in the case of the factor model, while $\lambda$ remains non-zero for negative values of the correlation coefficient for bivariate Student's variables.

If $Y$ and $\epsilon$ have different numbers $\nu_{Y}$ and $\nu_{\epsilon}$ of degrees of freedom, two cases occur. For $\nu_{Y}<\nu_{\epsilon}, \epsilon$ is negligible asymptotically and $\lambda=1$. For $\nu_{Y}>\nu_{\epsilon}, X$ becomes identical to $\epsilon$ because $Y$ is negligible asymptotically. Thus $X$ and $Y$ have the same tail-dependence as $\epsilon$ and $Y$, which is 0 by construction.

\subsubsection{General case}

A general result concerning the tail dependence generated by factor models for every kind of factor and noise distributions has recently been established by [Malevergne and Sornette (2002)]. It has been proved that the coefficient of (upper) tail dependence between $X$ and $Y$ is given by

$$
\lambda=\int_{\max \left\{1, \frac{l}{\alpha}\right\}}^{\infty} d x f(x),
$$

where, provided that they exist,

$$
\begin{aligned}
l & =\lim _{u \rightarrow 1} \frac{F_{X}^{-1}(u)}{F_{Y}^{-1}(u)}, \\
f(x) & =\lim _{t \rightarrow \infty} \frac{t \cdot P_{Y}(t \cdot x)}{\bar{F}_{Y}(t)} .
\end{aligned}
$$

In the framework of this general theorem $(\sqrt{36})$, the previous result (34) is a special case which can be easily retrieved.

As a direct consequence, one can show that any rapidly varying factor leads to a vanishing coefficient of tail dependence, whatever the distribution of the idiosyncratic noise may be. On the contrary, regularly vaying 
factors lead to a tail dependence, provided that the distribution of the idiosycratic noise does not become fatter-tailed than the factor distribution. One can thus conclude that, in order to generate tail dependence, the factor must have a sufficiently 'wild' distribution.

\subsection{Estimation of the coefficient of tail dependence}

It would seem that the coefficient of tail dependence could provide a useful measure of the extreme dependence between two random variables. Indeed, it could be an interesting quantity to study for risk management purposes since it gives directly the probabilty that an asset suffers a large loss assuming that a large loss occured for another asset. It is also important for contagion problems. Indeed, the evolution of the coefficient of tail dependence with the volatility of the markets could be a very significant indicator of possible contagions between these markets.

Unfortunately, the empirical estimation of the coefficient of tail dependence is a strenuous task, and to our knowledge, there is not yet any reliable estimator. A direct estimation of the conditional probability $\operatorname{Pr}\left\{X>F_{X}^{-1}(u) \mid Y>F_{Y}^{-1}(u)\right\}$, which should tend to $\lambda$ when $u \rightarrow 1$ is impossible to put in practice due to the combination of the curse of dimensionality and the drastic decrease of the number of realisations as $u$ become close to one.

A better approach consists in using kernel estimators, which generally provide smooth and accurate estimators KKulpa (1999), Li et al. (1998), Scaillet (2000)]. However, these smooth estimators lead to differentiable estimated copulas which have automatically vanishing tail dependence. Indeed, in order to obtain a non-vanishing coefficient of tail dependence, it is necessary for the corresponding copula to be non-differentiable at the point $(1,1)$ (or at $(0,0)$ ).

An alternative is the parametric approach. One can choose to model dependence via a specific copula, and thus to determine the associated tail dependence [Longin and Solnik (2001), Malevergne and Sornette (2001), Patton (2001)]. The problem of such a method is that the choice of the parameterization of the copula amounts to choose a priori whether or not the data presents tail dependence.

An improvement in the estimation of tail dependence has been obtained recently by using the semi-parametric approach of [Malevergne and Sornette (2002)]. This approach is based on the existence of a representation of dependence in terms of a factor model. In this approach, the estimation of the tail dependence relies solely on the estimation of the marginal distributions, a significantly easier task.

\section{Summary and Discussion}

Table 1 summarizes the asymptotic dependences for large $v$ and $u$ of the signed conditional correlation coefficient $\rho_{v}^{+}$, the unsigned conditional correlation coefficient $\rho_{v}^{s}$ and the correlation coefficient $\rho_{u}$ conditioned on both variables for the bivariate Gaussian, the Student's model, the Gaussian factor model and the Student's factor model. Our results provide a quantitative proof that conditioning on exceedance leads to conditional correlation coefficients that may be very different from the unconditional correlation. This provides a straightforward mechanism for fluctuations or changes of correlations, based on fluctuations of volatility or changes of trends. In other words, the many reported variations of correlation structure may be in large part attributed to changes in volatility. For instance, our result (13) for the Student's factor model explains the Monte-Carlo and empirical results [Cizeau et al. (2001)] that the unsigned conditional correlation $\rho_{v}^{s}$ increases with the exceedance level $v$.

We also suggest that the distinct dependences as a function of exceedance $v$ and $u$ of the conditional correla- 
tion coefficients may offer novel tools for characterizing the statistical multivariate distributions of extreme events. Since their direct characterization is in general restricted by the curse of dimensionality and the scarsity of data, the conditional correlation coefficients provide reduced robust statistics which can be estimated with reasonable accuracy and reliability.

Table 1 gives the asymptotic values of $\rho_{v}^{+}, \rho_{v}^{s}$ and $\rho_{u}$ for $v \rightarrow+\infty$ and $u \rightarrow \infty$ in order to compare them with the tail-dependence $\lambda$.

These two tables only scratch the surface of the rich sets of measures of tail and extreme dependences. We have shown that complete independence (factorization of the bivariate distribution) implies no tail dependence $\lambda=0$. But $\lambda=0$ does not imply factorization. Conversely, tail dependence $\lambda>0$ implies absence of factorization. But $\lambda>0$ does not imply necessarily that the conditional correlation coefficients $\rho_{v=\infty}^{+}$ and $\rho_{v=\infty}^{s}$ are non-zero.

Note that the examples of Table 2 are such that $\lambda=0$ seems to go hand-in-hand with $\rho_{v \rightarrow \infty}^{+}=0$. However, the logical implication $(\lambda=0) \Rightarrow\left(\rho_{v \rightarrow \infty}^{+}=0\right)$ does not hold in general. A counter example is offered by the Student's factor model in the case where $\nu_{Y}>\nu_{\epsilon}$ (the tail of the distribution of the idiosynchratic noise is fatter than that of the distribution of the factor). In this case, $X$ and $Y$ have the same tail-dependence as $\epsilon$ and $Y$, which is 0 by construction. But, $\rho_{v=\infty}^{+}$and $\rho_{v=\infty}^{s}$ are both 1 because a large $Y$ almost always gives a large $X$ and the simultaneous occurrence of a large $Y$ and a large $\epsilon$ can be neglected. The reason for this absence of tail dependence (in the sense of $\lambda$ ) coming together with asymptotically strong conditional correlation coefficients stems from two facts:

- first, the conditional correlation coefficients put much less weight on the extreme tails that the taildependence parameter $\lambda$. In other words, $\rho_{v=\infty}^{+}$and $\rho_{v=\infty}^{s}$ are sensitive to the marginals, i.e., there are determined by the full bivariate distribution, while, as we said, $\lambda$ is a pure copula property independent of the marginals. Since $\rho_{v=\infty}^{+}$and $\rho_{v=\infty}^{s}$ are measures of tail dependence weighted by the specific shapes of the marginals, it is natural that they may behave differently.

- Secondly, the tail dependence $\lambda$ probes the extreme dependence property of the original copula of the random variables $X$ and $Y$. On the contrary, when conditioning on $Y$, one changes the copula of $X$ and $Y$, so that the extreme dependence properties investigated by the conditional correlations are not exactly those of the original copula. This last remark explains clearly why we observe what [Boyer et al. (1997)] call a "bias" in the conditional correlations. Indeed, changing the dependence between two random variables obviously leads to change their correlations.

The consequences are potentially of importance. In such a situation, one measure $(\lambda)$ would conclude on asymptotic tail-independence while the other measures $\rho_{v=\infty}^{+}$and $\rho_{v=\infty}^{s}$ would conclude the opposite. Thus, before concluding on a change in the dependence structure with respect to a given parameter - the volatility or the trend, for instance - one should check that this change does not result from the tool used to probe the dependence. In this respect, as recently stressed by [Forbes and Rigobon (2001)], many previous contagion studies may be unreliable.

These questions related to state-varying-dependence are also very important for practical applications. As stressed by Ang and Bekaert (2000), Ang and Chen (2001)] for instance, the optimal portfolio will also become state-dependent, and neglecting this point can lead to very inefficient asset allocation. We would like to add that, for this purpose, the time scales involved - i.e., the investment horizon -will determine which measure is most appropriate. 


\section{A Conditional correlation coefficient for Gaussian variables}

Let us consider a pair of Normal random variables $(X, Y) \sim \mathcal{N}(0, \Sigma)$ where $\Sigma$ is their covariance matrix with unconditional correlation coefficient $\rho$. Without loss of generality, and for simplicity, we shall assume $\Sigma$ with unit unconditional variances.

\section{A.1 Conditioning on $Y$ larger than $v$}

Given a conditioning set $\mathcal{A}=[v,+\infty), v \in \mathbb{R}_{+}, \rho_{\mathcal{A}}=\rho_{v}^{+}$is the correlation coefficient conditioned on $Y$ larger than $v$ :

$$
\rho_{v}^{+}=\frac{\rho}{\sqrt{\rho^{2}+\frac{1-\rho^{2}}{\operatorname{Var}(Y \mid Y>v)}}} .
$$

We start with the calculation of the first and the second moment of $Y$ conditioned on $Y$ larger than $v$ :

$$
\begin{aligned}
\mathrm{E}(Y \mid Y>v) & =\frac{\sqrt{2}}{\sqrt{\pi} e^{\frac{v^{2}}{2}} \operatorname{erfc}\left(\frac{v}{\sqrt{2}}\right)}=v+\frac{1}{v}-\frac{2}{v^{3}}+\mathcal{O}\left(\frac{1}{v^{5}}\right), \\
\mathrm{E}\left(Y^{2} \mid Y>v\right) & =1+\frac{\sqrt{2} v}{\sqrt{\pi} e^{\frac{v^{2}}{2}} \operatorname{erfc}\left(\frac{v}{\sqrt{2}}\right)}=v^{2}+2-\frac{2}{v^{2}}+\mathcal{O}\left(\frac{1}{v^{4}}\right),
\end{aligned}
$$

which allows us to obtain the variance of $Y$ conditioned on $Y$ larger than $v$ :

$$
\operatorname{Var}(Y \mid Y>v)=1+\frac{\sqrt{2} v}{\sqrt{\pi} e^{\frac{v^{2}}{2}} \operatorname{erfc}\left(\frac{v}{\sqrt{2}}\right)}-\left(\frac{\sqrt{2}}{\sqrt{\pi} e^{\frac{v^{2}}{2}} \operatorname{erfc}\left(\frac{v}{\sqrt{2}}\right)}\right)^{2}=\frac{1}{v^{2}}+\mathcal{O}\left(\frac{1}{v^{4}}\right)
$$

which, for large $v$, yields:

$$
\rho_{v}^{+} \sim_{v \rightarrow \infty} \frac{\rho}{\sqrt{1-\rho^{2}}} \cdot \frac{1}{v}
$$

\section{A.2 Conditioning on $|Y|$ larger than $v$}

Given a conditioning set $\mathcal{A}=(-\infty,-v] \cup[v,+\infty), v \in \mathbb{R}_{+}, \rho_{\mathcal{A}}=\rho_{v}^{s}$ is the correlation coefficient conditioned on $|Y|$ larger than $v$ :

$$
\rho_{v}^{s}=\frac{\rho}{\sqrt{\rho^{2}+\frac{1-\rho^{2}}{\operatorname{Var}(Y|| Y \mid>v)}}}
$$

The first and second moment of $Y$ conditioned on $|Y|$ larger than $v$ can be easily calculated:

$$
\begin{aligned}
\mathrm{E}(Y|| Y \mid>v) & =0, \\
\mathrm{E}\left(Y^{2}|| Y \mid>v\right) & =1+\frac{\sqrt{2} v}{\sqrt{\pi} e^{\frac{v^{2}}{2}} \operatorname{erfc}\left(\frac{v}{\sqrt{2}}\right)}=v^{2}+2-\frac{2}{v^{2}}+\mathcal{O}\left(\frac{1}{v^{4}}\right) .
\end{aligned}
$$

Expression (46) is the same as (42) as it should. This gives the following conditional variance:

$$
\operatorname{Var}(Y|| Y \mid>v)=1+\frac{\sqrt{2} v}{\sqrt{\pi} e^{\frac{v^{2}}{2}} \operatorname{erfc}\left(\frac{v}{\sqrt{2}}\right)}=v^{2}+2+\mathcal{O}\left(\frac{1}{v^{2}}\right)
$$


and finally yields, for large $v$,

$$
\rho_{v}^{s} \sim_{v \rightarrow \infty} \frac{\rho}{\sqrt{\rho^{2}+\frac{1-\rho^{2}}{2+v^{2}}}} \sim_{v \rightarrow \infty} 1-\frac{1}{2} \frac{1-\rho^{2}}{\rho^{2}} \frac{1}{v^{2}} .
$$

\section{A.3 Conditioning on both $X$ and $Y$ larger than $u$}

By definition, the conditional correlation coefficient $\rho_{u}$, conditioned on both $X$ and $Y$ larger than $u$, is

$$
\begin{aligned}
\rho_{u} & =\frac{\operatorname{Cov}[X, Y \mid X>u, Y>u]}{\sqrt{\operatorname{Var}[X \mid X>u, Y>u]} \sqrt{\operatorname{Var}[Y \mid X>u, Y>u]}}, \\
& =\frac{m_{11}-m_{10} \cdot m_{01}}{\sqrt{m_{20}-m_{10}^{2}} \sqrt{m_{02}-m_{01}^{2}}},
\end{aligned}
$$

where $m_{i j}$ denotes $\mathrm{E}\left[X^{i} \cdot Y^{j} \mid X>u, Y>u\right]$.

Using the proposition A.1 of [Ang and Chen (2001)] or the expressions in [Johnson and Kotz (1972), p 113], we can assert that

$$
\begin{aligned}
& m_{10} L(u, u ; \rho)=(1+\rho) \phi(u)\left[1-\Phi\left(\sqrt{\frac{1-\rho}{1+\rho}} u\right)\right] \\
& m_{20} L(u, u ; \rho)=\left(1+\rho^{2}\right) u \phi(u)\left[1-\Phi\left(\sqrt{\frac{1-\rho}{1+\rho}} u\right)\right]+\frac{\rho \sqrt{1-\rho^{2}}}{\sqrt{2 \pi}} \phi\left(\sqrt{\frac{2}{1+\rho}} u\right)+L(u, u ; \rho), \\
& m_{11} L(u, u ; \rho)=2 \rho u \phi(u)\left[1-\Phi\left(\sqrt{\frac{1-\rho}{1+\rho}} u\right)\right]+\frac{\sqrt{1-\rho^{2}}}{\sqrt{2 \pi}} \phi\left(\sqrt{\frac{2}{1+\rho}} u\right)+\rho L(u, u ; \rho),
\end{aligned}
$$

where $L(\cdot, \cdot ; \cdot)$ denotes the bivariate Gaussian survival (or complementary cumulative) distribution:

$$
L(h, k ; \rho)=\frac{1}{2 \pi \sqrt{1-\rho^{2}}} \int_{h}^{\infty} d x \int_{k}^{\infty} d y \exp \left(-\frac{1}{2} \frac{x^{2}-2 \rho x y+y^{2}}{1-\rho^{2}}\right),
$$

$\phi(\cdot)$ is the Gaussian density:

$$
\phi(x)=\frac{1}{\sqrt{2 \pi}} e^{-\frac{x^{2}}{2}}
$$

and $\Phi(\cdot)$ is the cumulative Gaussian distribution:

$$
\Phi(x)=\int_{-\infty}^{x} d u \phi(u) .
$$

\section{A.3.1 Asymptotic behavior of $L(u, u ; \rho)$}

We focus on the asymptotic behavior of

$$
L(u, u ; \rho)=\frac{1}{2 \pi \sqrt{1-\rho^{2}}} \int_{u}^{\infty} d x \int_{u}^{\infty} d y \exp \left(-\frac{1}{2} \frac{x^{2}-2 \rho x y+y^{2}}{1-\rho^{2}}\right),
$$

for large $u$. Performing the change of variables $x^{\prime}=x-u$ and $y^{\prime}=y-u$, we can write

$$
L(u, u ; \rho)=\frac{e^{-\frac{u^{2}}{1+\rho}}}{2 \pi \sqrt{1-\rho^{2}}} \int_{0}^{\infty} d x^{\prime} \int_{0}^{\infty} d y^{\prime} \exp \left(-u \frac{x^{\prime}+y^{\prime}}{1+\rho}\right) \exp \left(-\frac{1}{2} \frac{x^{\prime 2}-2 \rho x^{\prime} y^{\prime}+y^{\prime 2}}{1-\rho^{2}}\right)
$$


Using the fact that

$$
\exp \left(-\frac{1}{2} \frac{x^{\prime 2}-2 \rho x^{\prime} y^{\prime}+y^{\prime 2}}{1-\rho^{2}}\right)=1-\frac{x^{\prime 2}-2 \rho x^{\prime} y^{\prime}+y^{\prime 2}}{2\left(1-\rho^{2}\right)}+\frac{\left(x^{\prime 2}-2 \rho x^{\prime} y^{\prime}+y^{\prime 2}\right)^{2}}{8\left(1-\rho^{2}\right)^{2}}-\frac{\left(x^{\prime 2}-2 \rho x^{\prime} y^{\prime}+y^{\prime 2}\right)^{3}}{48\left(1-\rho^{2}\right)^{3}}+\cdots,
$$

and applying theorem 3.1.1 in [Jensen (1995), p 58] (Laplace's method), equations (58) and (59) yield

$$
\begin{aligned}
& L(u, u ; \rho)=\frac{(1+\rho)^{2}}{2 \pi \sqrt{1-\rho^{2}}} \cdot \frac{e^{-\frac{u^{2}}{1+\rho}}}{u^{2}}\left[1-\frac{(2-\rho)(1+\rho)}{1-\rho} \cdot \frac{1}{u^{2}}+\frac{\left(2 \rho^{2}-6 \rho+7\right)(1+\rho)^{2}}{(1-\rho)^{2}} \cdot \frac{1}{u^{4}}\right. \\
& \left.-3 \frac{\left(12-13 \rho+8 \rho^{2}-2 \rho^{3}\right)(1+\rho)^{3}}{(1-\rho)^{3}} \cdot \frac{1}{u^{6}}+\mathcal{O}\left(\frac{1}{u^{8}}\right)\right],
\end{aligned}
$$

and

$$
\begin{aligned}
1 / L(u, u ; \rho)=\frac{2 \pi u^{2} \sqrt{1-\rho^{2}}}{(1+\rho)^{2}} \cdot e^{\frac{u^{2}}{1+\rho}}[ & 1+\frac{(2-\rho)(1+\rho)}{1-\rho} \cdot \frac{1}{u^{2}}-\frac{\left.3-2 \rho+\rho^{2}\right)(1+\rho)^{2}}{(1-\rho)^{2}} \cdot \frac{1}{u^{4}} \\
& \left.+\frac{\left(16-13 \rho+10 \rho^{2}-3 \rho^{3}\right)(1+\rho)^{3}}{(1-\rho)^{3}} \cdot \frac{1}{u^{6}}+\mathcal{O}\left(\frac{1}{u^{8}}\right)\right] .
\end{aligned}
$$

\section{A.3.2 Asymptotic behavior of the first moment $m_{10}$}

The first moment $m_{10}=\mathrm{E}[X \mid X>u, Y>u]$ is given by (51). For large $u$,

$$
\begin{aligned}
1-\Phi\left(\sqrt{\frac{1-\rho}{1+\rho}} u\right)= & \frac{1}{2} \operatorname{erfc}\left(\sqrt{\left.\frac{1-\rho}{2(1+\rho)} u\right)}\right. \\
= & \sqrt{\frac{1+\rho}{1-\rho}} \frac{e^{-\frac{1-\rho}{2(1+\rho)}} u^{2}}{\sqrt{2 \pi} u}\left[1-\frac{1+\rho}{1-\rho} \cdot \frac{1}{u^{2}}+3\left(\frac{1+\rho}{1-\rho}\right)^{2} \cdot \frac{1}{u^{4}}\right. \\
& \left.-15\left(\frac{1+\rho}{1-\rho}\right)^{3} \cdot \frac{1}{u^{6}}+\mathcal{O}\left(\frac{1}{u^{8}}\right)\right],
\end{aligned}
$$

so that multiplying by $(1+\rho) \phi(u)$, we obtain

$m_{10} L(u, u ; \rho)=\frac{(1+\rho)^{2}}{\sqrt{1-\rho^{2}}} \frac{e^{-\frac{u^{2}}{1+\rho}}}{2 \pi u}\left[1-\frac{1+\rho}{1-\rho} \cdot \frac{1}{u^{2}}+3\left(\frac{1+\rho}{1-\rho}\right)^{2} \cdot \frac{1}{u^{4}}-15\left(\frac{1+\rho}{1-\rho}\right)^{3} \cdot \frac{1}{u^{6}}+\mathcal{O}\left(\frac{1}{u^{8}}\right)\right]$.

Using the result given by equation (60), we can conclude that

$$
m_{10}=u+(1+\rho) \cdot \frac{1}{u}-\frac{(1+\rho)^{2}(2-\rho)}{(1-\rho)} \cdot \frac{1}{u^{3}}+\frac{\left(10-8 \rho+3 \rho^{2}\right)(1+\rho)^{3}}{(1-\rho)^{2}} \cdot \frac{1}{u^{5}}+\mathcal{O}\left(\frac{1}{u^{7}}\right) .
$$

In the sequel, we will also need the behavior of $m_{10}{ }^{2}$ :

$$
m_{10}^{2}=u^{2}+2(1+\rho)-\frac{(1+\rho)^{2}(3-\rho)}{(1-\rho)} \cdot \frac{1}{u^{2}}+2 \frac{\left(8-5 \rho+2 \rho^{2}\right)(1+\rho)^{3}}{(1-\rho)^{2}} \cdot \frac{1}{u^{4}}+\mathcal{O}\left(\frac{1}{u^{6}}\right) .
$$




\section{A.3.3 Asymptotic behavior of the second moment $m_{20}$}

The second moment $m_{20}=\mathrm{E}\left[X^{2} \mid X>u, Y>u\right]$ is given by expression (52). The first term in the right hand side of (52) yields

$$
\begin{array}{r}
\left(1+\rho^{2}\right) u \phi(u)\left[1-\Phi\left(\sqrt{\frac{1-\rho}{1+\rho}} u\right)\right]=\left(1+\rho^{2}\right) \sqrt{\frac{1+\rho}{1-\rho}} \frac{e^{-\frac{u^{2}}{1+\rho}}}{2 \pi}\left[1-\frac{1+\rho}{1-\rho} \cdot \frac{1}{u^{2}}+3\left(\frac{1+\rho}{1-\rho}\right)^{2} \cdot \frac{1}{u^{4}}\right. \\
\left.-15\left(\frac{1+\rho}{1-\rho}\right)^{3} \cdot \frac{1}{u^{6}}+\mathcal{O}\left(\frac{1}{u^{8}}\right)\right]
\end{array}
$$

while the second term gives

$$
\frac{\rho \sqrt{1-\rho^{2}}}{\sqrt{2 \pi}} \phi\left(\sqrt{\frac{2}{1+\rho}} u\right)=\rho \sqrt{1-\rho^{2}} \frac{e^{-\frac{u^{2}}{1+\rho}}}{2 \pi} .
$$

Putting these two expressions together and factorizing the term $(1+\rho) /\left(1+\rho^{2}\right)$ allows us to obtain

$$
\begin{array}{r}
m_{20} L(u, u ; \rho)=\frac{(1+\rho)^{2}}{\sqrt{1-\rho^{2}}} \frac{e^{-\frac{u^{2}}{1+\rho}}}{2 \pi}\left[1-\frac{1+\rho^{2}}{1-\rho} \cdot \frac{1}{u^{2}}+3 \frac{\left(1+\rho^{2}\right)(1+\rho)}{(1-\rho)^{2}} \cdot \frac{1}{u^{4}}\right. \\
\left.-15 \frac{\left(1+\rho^{2}\right)(1+\rho)^{2}}{(1-\rho)^{3}} \cdot \frac{1}{u^{6}}+\mathcal{O}\left(\frac{1}{u^{8}}\right)\right]+L(u, u ; \rho),
\end{array}
$$

which finally yields

$$
m_{20}=u^{2}+2(1+\rho)-2 \frac{(1+\rho)^{2}}{1-\rho} \cdot \frac{1}{u^{2}}+2 \frac{\left(5+4 \rho+\rho^{3}\right)(1+\rho)^{2}}{(1-\rho)^{2}} \frac{1}{u^{4}}+\mathcal{O}\left(\frac{1}{u^{6}}\right) .
$$

\section{A.3.4 Asymptotic behavior of the cross moment $m_{11}$}

The cross moment $m_{11}=\mathrm{E}[X \cdot Y \mid X>u, Y>u]$ is given by expression (53). The first and second terms in the right hand side of (53) respectively give

$$
\begin{aligned}
& 2 \rho u \phi(u)[1-\Phi(u)]=2 \rho \sqrt{\frac{1+\rho}{1-\rho}} \frac{e^{-\frac{u^{2}}{1+\rho}}}{2 \pi}[1-\frac{1+\rho}{1-\rho} \cdot \frac{1}{u^{2}}+3\left(\frac{1+\rho}{1-\rho}\right)^{2} \cdot \frac{1}{u^{4}} \\
&\left.-15\left(\frac{1+\rho}{1-\rho}\right)^{3} \cdot \frac{1}{u^{6}}+\mathcal{O}\left(\frac{1}{u^{8}}\right)\right], \\
& \frac{\sqrt{1-\rho^{2}}}{\sqrt{2 \pi}} \phi\left(\sqrt{\left.\frac{2}{1+\rho} u\right)}=\sqrt{1-\rho^{2}} \frac{e^{-\frac{u^{2}}{1+\rho}}}{2 \pi}\right.
\end{aligned}
$$

which, after factorization by $(1+\rho) / \rho$, yields

$$
\begin{array}{r}
m_{11} L(u, u ; \rho)=\frac{(1+\rho)^{2}}{\sqrt{1-\rho^{2}}} \frac{e^{-\frac{u^{2}}{1+\rho}}}{2 \pi}\left[1-2 \frac{\rho}{1-\rho} \cdot \frac{1}{u^{2}}+6 \frac{\rho(1+\rho)}{(1-\rho)^{2}} \cdot \frac{1}{u^{4}}\right. \\
\left.-30 \frac{\rho(1+\rho)^{2}}{(1-\rho)^{3}} \cdot \frac{1}{u^{6}}+\mathcal{O}\left(\frac{1}{u^{8}}\right)\right]+\rho L(u, u ; \rho),
\end{array}
$$

and finally

$$
m_{11}=u^{2}+2(1+\rho)-\frac{(1+\rho)^{2}(3-\rho)}{(1-\rho)} \cdot \frac{1}{u^{2}}+\frac{\left(16-9 \rho+3 \rho^{2}\right)(1+\rho)^{3}}{(1-\rho)^{2}} \cdot \frac{1}{u^{4}}+\mathcal{O}\left(\frac{1}{u^{6}}\right) .
$$




\section{A.3.5 Asymptotic behavior of the correlation coefficient}

The conditional correlation coefficient conditioned on both $X$ and $Y$ larger than $u$ is defined by (50). Using the symmetry between $X$ and $Y$, we have $m_{10}=m_{01}$ and $m_{20}=m_{02}$, which allows us to rewrite (50) as follows

$$
\rho_{u}=\frac{m_{11}-m_{10}^{2}}{m_{20}-m_{10}^{2}} .
$$

Putting together the previous results, we have

$$
\begin{aligned}
& m_{20}-m_{10}^{2}=\frac{(1+\rho)^{2}}{u^{2}}-2 \frac{\left(4-\rho+3 \rho^{2}+3 \rho^{3}\right)(1+\rho)^{2}}{1-\rho} \cdot \frac{1}{u^{4}}+\mathcal{O}\left(\frac{1}{u^{6}}\right), \\
& m_{11}-m_{10}^{2}=\rho \frac{(1+\rho)^{3}}{1-\rho} \cdot \frac{1}{u^{4}}+\mathcal{O}\left(\frac{1}{u^{6}}\right)
\end{aligned}
$$

which proves that

$$
\rho_{u}=\rho \frac{1+\rho}{1-\rho} \cdot \frac{1}{u^{2}}+\mathcal{O}\left(\frac{1}{u^{4}}\right) \text { and } \rho \in[-1,1)
$$




\section{B Conditional correlation coefficient for Student's variables}

\section{B.1 Proposition}

Let us consider a pair of Student's random variables $(X, Y)$ with $\nu$ degrees of freedom and unconditional correlation coefficient $\rho$. Let $\mathcal{A}$ be a subset of $\mathbb{R}$ such that $\operatorname{Pr}\{Y \in \mathcal{A}\}>0$. The correlation coefficient of $(X, Y)$, conditioned on $Y \in \mathcal{A}$ defined by

$$
\rho_{\mathcal{A}}=\frac{\operatorname{Cov}(X, Y \mid Y \in \mathcal{A})}{\sqrt{\operatorname{Var}(X \mid Y \in \mathcal{A})} \sqrt{\operatorname{Var}(Y \mid Y \in \mathcal{A})}} .
$$

can be expressed as

$$
\rho_{\mathcal{A}}=\frac{\rho}{\sqrt{\rho^{2}+\frac{\mathrm{E}\left[\mathrm{E}\left(x^{2} \mid Y\right)-\rho^{2} Y^{2} \mid Y \in \mathcal{A}\right]}{\operatorname{Var}(Y \mid Y \in \mathcal{A})}}},
$$

with

$$
\operatorname{Var}(Y \mid Y \in \mathcal{A})=\nu\left[\frac{\nu-1}{\nu-2} \cdot \frac{\operatorname{Pr}\left\{\sqrt{\frac{\nu}{\nu-2}} Y \in \mathcal{A} \mid \nu-2\right\}}{\operatorname{Pr}\{Y \in \mathcal{A} \mid \nu\}}-1\right]-\left[\frac{\int_{y \in \mathcal{A}} d y y \cdot t_{y}(y)}{\operatorname{Pr}\{Y \in \mathcal{A} \mid \nu\}}\right]^{2}
$$

and

$$
\mathrm{E}\left[\mathrm{E}\left(X^{2} \mid Y\right)-\rho^{2} Y^{2} \mid Y \in \mathcal{A}\right]=\left(1-\rho^{2}\right) \frac{\nu}{\nu-2} \cdot \frac{\operatorname{Pr}\left\{\sqrt{\frac{\nu}{\nu-2}} Y \in \mathcal{A} \mid \nu-2\right\}}{\operatorname{Pr}\{Y \in \mathcal{A} \mid \nu\}} .
$$

\section{B.2 Proof of the proposition}

Let the variables $X$ and $Y$ have a multivariate Student's distribution with $\nu$ degrees of freedom and a correlation coefficient $\rho$ :

$$
\begin{aligned}
P_{X Y}(x, y) & =\frac{\Gamma\left(\frac{\nu+2}{2}\right)}{\nu \pi \Gamma\left(\frac{\nu+1}{2}\right) \sqrt{1-\rho^{2}}}\left(1+\frac{x^{2}-2 \rho x y+y^{2}}{\nu\left(1-\rho^{2}\right)}\right)^{-\frac{\nu+2}{2}}, \\
& =\left(\frac{\nu+1}{\nu+y^{2}}\right)^{1 / 2} \frac{1}{\sqrt{1-\rho^{2}}} t_{\nu}(y) \cdot t_{\nu+1}\left[\left(\frac{\nu+1}{\nu+y^{2}}\right)^{1 / 2} \frac{x-\rho y}{\sqrt{1-\rho^{2}}}\right],
\end{aligned}
$$

where $t_{\nu}(\cdot)$ denotes the univariate Student's density with $\nu$ degrees of freedom

$$
t_{\nu}(x)=\frac{\Gamma\left(\frac{\nu+1}{2}\right)}{\Gamma\left(\frac{\nu}{2}\right)(\nu \pi)^{1 / 2}} \cdot \frac{1}{\left(1+\frac{x^{2}}{\nu}\right)^{\frac{\nu+1}{2}}}=\frac{C_{\nu}}{\left(1+\frac{x^{2}}{\nu}\right)^{\frac{\nu+1}{2}}} .
$$

Let us evaluate $\operatorname{Cov}(X, Y \mid Y \in \mathcal{A})$ :

$$
\begin{aligned}
\operatorname{Cov}(X, Y \mid Y \in \mathcal{A}) & =\mathrm{E}(X \cdot Y \mid Y \in \mathcal{A})-\mathrm{E}(X \mid Y \in \mathcal{A}) \cdot \mathrm{E}(Y \mid Y \in \mathcal{A}) \\
& =\mathrm{E}(\mathrm{E}(X \mid Y) \cdot Y \mid Y \in \mathcal{A})-\mathrm{E}(\mathrm{E}(X \mid Y) \mid Y \in \mathcal{A}) \cdot \mathrm{E}(Y \mid Y \in \mathcal{A})
\end{aligned}
$$

As it can be seen in equation (84), $\mathrm{E}(X \mid Y)=\rho Y$, which gives

$$
\begin{aligned}
\operatorname{Cov}(X, Y \mid Y \in \mathcal{A}) & =\rho \cdot \mathrm{E}\left(Y^{2} \mid Y \in \mathcal{A}\right)-\rho \cdot \mathrm{E}(Y \mid Y \in \mathcal{A})^{2}, \\
& =\rho \cdot \operatorname{Var}(Y \mid Y \in \mathcal{A}) .
\end{aligned}
$$


Thus, we have

$$
\rho_{\mathcal{A}}=\rho \sqrt{\frac{\operatorname{Var}(Y \mid Y \in \mathcal{A})}{\operatorname{Var}(X \mid Y \in \mathcal{A})}} .
$$

Using the same method as for the calculation of $\operatorname{Cov}(X, Y \mid Y \in \mathcal{A})$, we find

$$
\begin{aligned}
\operatorname{Var}(X \mid Y \in \mathcal{A}) & \left.\left.=\mathrm{E}\left[\mathrm{E}\left(X^{2} \mid Y\right) \mid Y \in \mathcal{A}\right)\right]-\mathrm{E}[\mathrm{E}(X \mid Y) \mid Y \in \mathcal{A})\right]^{2} \\
& \left.=\mathrm{E}\left[\mathrm{E}\left(X^{2} \mid Y\right) \mid Y \in \mathcal{A}\right)\right]-\rho^{2} \cdot \mathrm{E}[Y \mid Y \in \mathcal{A}]^{2} \\
& \left.=\mathrm{E}\left[\mathrm{E}\left(X^{2} \mid Y\right)-\rho^{2} Y^{2} \mid Y \in \mathcal{A}\right)\right]-\rho^{2} \cdot \operatorname{Var}[Y \mid Y \in \mathcal{A}]
\end{aligned}
$$

which yields

$$
\rho_{\mathcal{A}}=\frac{\rho}{\sqrt{\rho^{2}+\frac{\mathrm{E}\left[\mathrm{E}\left(x^{2} \mid Y\right)-\rho^{2} Y^{2} \mid Y \in \mathcal{A}\right]}{\operatorname{Var}(Y \mid Y \in \mathcal{A})}}},
$$

as asserted in $(80)$.

To go one step further, we have to evaluate the three terms $\mathrm{E}(Y \mid Y \in \mathcal{A}), \mathrm{E}\left(Y^{2} \mid Y \in \mathcal{A}\right)$, and $\mathrm{E}\left[\mathrm{E}\left(X^{2} \mid Y\right) \mid Y \in \mathcal{A}\right]$.

The first one is trivial to calculate :

$$
\mathrm{E}(Y \mid Y \in \mathcal{A})=\frac{\int_{y \in \mathcal{A}} d y y \cdot t_{y}(y)}{\operatorname{Pr}\{Y \in \mathcal{A} \mid \nu\}} .
$$

The second one gives

$$
\begin{aligned}
\mathrm{E}\left(Y^{2} \mid Y \in \mathcal{A}\right) & =\frac{\int_{y \in \mathcal{A}} d y y^{2} \cdot t_{y}(y)}{\operatorname{Pr}\{Y \in \mathcal{A} \mid \nu\}} \\
& =\nu\left[\frac{\nu-1}{\nu-2} \cdot \frac{\operatorname{Pr}\left\{\sqrt{\frac{\nu}{\nu-2}} Y \in \mathcal{A} \mid \nu-2\right\}}{\operatorname{Pr}\{Y \in \mathcal{A} \mid \nu\}}-1\right],
\end{aligned}
$$

so that

$$
\operatorname{Var}(Y \mid Y \in \mathcal{A})=\nu\left[\frac{\nu-1}{\nu-2} \cdot \frac{\operatorname{Pr}\left\{\sqrt{\frac{\nu}{\nu-2}} Y \in \mathcal{A} \mid \nu-2\right\}}{\operatorname{Pr}\{Y \in \mathcal{A} \mid \nu\}}-1\right]-\left[\frac{\int_{y \in \mathcal{A}} d y y \cdot t_{y}(y)}{\operatorname{Pr}\{Y \in \mathcal{A} \mid \nu\}}\right]^{2}
$$

To calculate the third term, we first need to evaluate $\mathrm{E}\left(X^{2} \mid Y\right)$. Using equation (84) and the results given in [Abramovitz and Stegun (1972)], we find

$$
\begin{aligned}
\mathrm{E}\left(X^{2} \mid Y\right) & =\int d x\left(\frac{\nu+1}{\nu+y^{2}}\right)^{1 / 2} \frac{x^{2}}{\sqrt{1-\rho^{2}}} \cdot t_{\nu+1}\left[\left(\frac{\nu+1}{\nu+y^{2}}\right)^{1 / 2} \frac{x-\rho y}{\sqrt{1-\rho^{2}}}\right] \\
& =\frac{\nu+y^{2}}{\nu-1}\left(1-\rho^{2}\right)-\rho^{2} y^{2}
\end{aligned}
$$

which yields

$$
\mathrm{E}\left[\mathrm{E}\left(X^{2} \mid Y\right)-\rho^{2} Y^{2} \mid Y \in \mathcal{A}\right]=\frac{\nu}{\nu-1}\left(1-\rho^{2}\right)+\frac{1-\rho^{2}}{\nu-1} \mathrm{E}\left[Y^{2} \mid Y \in \mathcal{A}\right],
$$


and applying the result given in eqation (98), we finally obtain

$$
\mathrm{E}\left[\mathrm{E}\left(X^{2} \mid Y\right)-\rho^{2} Y^{2} \mid Y \in \mathcal{A}\right]=\left(1-\rho^{2}\right) \frac{\nu}{\nu-2} \cdot \frac{\operatorname{Pr}\left\{\sqrt{\frac{\nu}{\nu-2}} Y \in \mathcal{A} \mid \nu-2\right\}}{\operatorname{Pr}\{Y \in \mathcal{A} \mid \nu\}},
$$

which concludes the proof.

\section{B.3 Conditioning on $Y$ larger than $v$}

The conditioning set is $\mathcal{A}=[v,+\infty)$, thus

$$
\begin{aligned}
\operatorname{Pr}\{Y \in \mathcal{A} \mid \nu\} & =\bar{T}_{\nu}(v)=\nu^{\frac{\nu-1}{2}} \frac{C_{\nu}}{v^{\nu}}+\mathcal{O}\left(v^{-(\nu+2)}\right), \\
\operatorname{Pr}\left\{\sqrt{\frac{\nu}{\nu-p}} Y \in \mathcal{A} \mid \nu-p\right\} & =\bar{T}_{\nu-p}\left(\sqrt{\frac{\nu-p}{\nu}} v\right)=\frac{\nu^{\frac{\nu-p}{2}}}{(\nu-p)^{\frac{1}{2}}} \frac{C_{\nu-p}}{v^{\nu-p}}+\mathcal{O}\left(v^{-(\nu-p+2)}\right), \\
\int_{y \in \mathcal{A}} d y y \cdot t_{y}(y) & =\sqrt{\frac{\nu}{\nu-2}} t_{\nu-2}\left(\sqrt{\frac{\nu-2}{\nu}} v\right)=\frac{\nu^{\frac{\nu}{2}}}{\sqrt{\nu-2}} \frac{C_{\nu-2}}{v^{\nu-1}}+\mathcal{O}\left(v^{-(\nu-3)}(106)\right.
\end{aligned}
$$

where $t_{\nu}(\cdot)$ and $\bar{T}_{\nu}(\cdot)$ denote respectively the density and the Student's survival distribution with $\nu$ degrees of freedom and $C_{\nu}$ is defined in (85).

Using equation (80), one can thus give the exact expression of $\rho_{v}^{+}$. Since it is very cumbersomme, we will not write it explicitely. We will only give the asymptotic expression of $\rho_{v}^{+}$. In this respect, we can show that

$$
\begin{aligned}
\operatorname{Var}(Y \mid Y \in \mathcal{A}) & =\frac{\nu}{(\nu-2)(\nu-1)^{2}} v^{2}+\mathcal{O}(1) \\
\mathrm{E}\left[\mathrm{E}\left(X^{2} \mid Y\right)-\rho^{2} Y^{2} \mid Y \in \mathcal{A}\right] & =\sqrt{\frac{\nu}{\nu-2} \frac{1-\rho^{2}}{\nu-1}} v^{2}+\mathcal{O}(1) .
\end{aligned}
$$

Thus, for large $v$,

$$
\rho_{v}^{+} \longrightarrow \frac{\rho}{\sqrt{\rho^{2}+(\nu-1) \sqrt{\frac{\nu-2}{\nu}}\left(1-\rho^{2}\right)}}
$$

\section{B.4 Conditioning on $|Y|$ larger than $v$}

The conditioning set is now $\mathcal{A}=(-\infty,-v] \cup[v,+\infty)$, with $v \in \mathbb{R}_{+}$. Thus, the right hand sides of equations (104) and (105) have to be multiplied by two while

$$
\int_{y \in \mathcal{A}} d y y \cdot t_{y}(y)=0
$$

for symmetry reasons. So the equation (108) still holds while

$$
\operatorname{Var}(Y \mid Y \in \mathcal{A})=\frac{\nu}{(\nu-2)} v^{2}+\mathcal{O}(1)
$$

Thus, for large $v$,

$$
\rho_{v}^{s} \longrightarrow \frac{\rho}{\sqrt{\rho^{2}+\frac{1}{(\nu-1)} \sqrt{\frac{\nu-2}{\nu}}\left(1-\rho^{2}\right)}} .
$$




\section{Proof of equation (III)}

We assume that $X$ and $Y$ are related by the equation

$$
X=\alpha Y+\epsilon,
$$

where $\alpha$ is a non random real coefficient and $\epsilon$ an idiosyncratic noise independent of $Y$, whose distribution is assumed to admit a moment of second order $\sigma_{\epsilon}^{2}$. Let us also denote by $\sigma_{y}^{2}$ the second moment of the variable $Y$.

We have

$$
\begin{aligned}
\operatorname{Cov}(X, Y \mid Y \in \mathcal{A}) & =\operatorname{Cov}(\alpha Y+\epsilon, Y \mid Y \in \mathcal{A}), \\
& =\alpha \operatorname{Var}(Y \mid Y \in \mathcal{A})+\operatorname{Cov}(\epsilon, Y \mid Y \in \mathcal{A}), \\
& =\alpha \operatorname{Var}(Y \mid Y \in \mathcal{A}),
\end{aligned}
$$

since $Y$ and $\epsilon$ are independent. We have also

$$
\begin{aligned}
\operatorname{Var}(X \mid Y \in \mathcal{A}) & =\alpha^{2} \operatorname{Var}(Y \mid Y \in \mathcal{A})+2 \operatorname{Cov}(\epsilon, Y \mid Y \in \mathcal{A})+\operatorname{Var}(\epsilon \mid Y \in \mathcal{A}) \\
& =\alpha^{2} \operatorname{Var}(Y \mid Y \in \mathcal{A})+\sigma_{\epsilon}^{2}
\end{aligned}
$$

where, again, we have used the independence of $Y$ and $\epsilon$. This allows us to write

$$
\begin{aligned}
\rho_{\mathcal{A}} & =\frac{\alpha \operatorname{Var}(Y \mid Y \in \mathcal{A})}{\sqrt{\operatorname{Var}(Y \mid Y \in \mathcal{A})\left(\alpha^{2} \operatorname{Var}(Y \mid Y \in \mathcal{A})+\sigma_{\epsilon}^{2}\right)}}, \\
& =\frac{\operatorname{sgn}(\alpha)}{\sqrt{1+\frac{\sigma_{\epsilon}^{2}}{\alpha^{2}} \cdot \frac{1}{\operatorname{Var}(Y \mid Y \in \mathcal{A})}}} .
\end{aligned}
$$

Since

$$
\rho=\frac{\operatorname{sgn}(\alpha)}{\sqrt{1+\frac{\sigma_{\epsilon}^{2}}{\alpha^{2}} \cdot \frac{1}{\operatorname{Var}(Y)}}},
$$

we finally obtain

$$
\rho_{\mathcal{A}}=\frac{\rho}{\sqrt{\rho^{2}+\left(1-\rho^{2}\right) \frac{\operatorname{Var}(y)}{\operatorname{Var}(y \mid y \in \mathcal{A})}}},
$$

which conclude the proof. 


\section{Conditional Spearman's rho}

The conditional Spearman's rho has been defined by

$$
\rho_{s}(\tilde{v})=\frac{\operatorname{Cov}(U, V \mid V \geq \tilde{v})}{\sqrt{\operatorname{Var}(U \mid V \geq \tilde{v}) \operatorname{Var}(V \mid \geq \tilde{v})}}
$$

We have

$$
\mathrm{E}[\cdot \mid V \geq \tilde{v}]=\frac{\int_{\tilde{v}}^{1} \int_{0}^{1} \cdot d C(u, v)}{\int_{\tilde{v}}^{1} \int_{0}^{1} d C(u, v)}=\frac{1}{1-\tilde{v}} \int_{\tilde{v}}^{1} \int_{0}^{1} \cdot d C(u, v),
$$

thus, performing a simple integration by parts, we obtain

$$
\begin{aligned}
\mathrm{E}[U \mid V \geq \tilde{v}] & =1+\frac{1}{1-\tilde{v}}\left[\int_{0}^{1} d u C(u, \tilde{v})-\frac{1}{2}\right], \\
\mathrm{E}[V \mid V \geq \tilde{v}] & =\frac{1+\tilde{v}}{2}, \\
\mathrm{E}\left[U^{2} \mid V \geq \tilde{v}\right] & =1+\frac{2}{1-\tilde{v}}\left[\int_{0}^{1} d u u C(u, \tilde{v})-\frac{1}{3}\right], \\
\mathrm{E}\left[V^{2} \mid V \geq \tilde{v}\right] & =\frac{\tilde{v}^{2}+\tilde{v}+1}{3}, \\
\mathrm{E}[U \cdot V \mid V \geq \tilde{v}] & =\frac{1+\tilde{v}}{2}+\frac{1}{1-\tilde{v}}\left[\int_{\tilde{v}}^{1} d v \int_{0}^{1} d u C(u, v)+\tilde{v} \int_{0}^{1} d u C(u, \tilde{v})-\frac{1}{2}\right],
\end{aligned}
$$

which yields

$$
\begin{aligned}
\operatorname{Cov}(U, V \mid V \geq \tilde{v})= & \frac{1}{1-\tilde{v}} \int_{\tilde{v}}^{1} d v \int_{0}^{1} d u C(u, v)-\frac{1}{2} \int_{0}^{1} d u C(u, \tilde{v})-\frac{1}{4} \\
\operatorname{Var}(U \mid V \geq \tilde{v})= & \frac{1-4 \tilde{v}}{12(1-\tilde{v})^{2}}+\frac{2}{1-\tilde{v}} \int_{0}^{1} d u u C(u, \tilde{v})+\frac{2 \tilde{v}-1}{(1-\tilde{v})^{2}} \int_{0}^{1} d u C(u, \tilde{v}) \\
& -\frac{1}{(1-\tilde{v})^{2}}\left(\int_{0}^{1} d u C(u, \tilde{v})\right)^{2} \\
\operatorname{Var}(V \mid V \geq \tilde{v})= & \frac{(1-\tilde{v})^{2}}{12}
\end{aligned}
$$

so that

$$
\rho_{s}(\tilde{v})=\frac{\frac{12}{1-\tilde{v}} \int_{\tilde{v}}^{1} d v \int_{0}^{1} d u C(u, v)-6 \int_{0}^{1} d u C(u, \tilde{v})-3}{\sqrt{1-4 \tilde{v}+24(1-\tilde{v}) \int_{0}^{1} d u u C(u, \tilde{v})+12(2 \tilde{v}-1) \int_{0}^{1} d u C(u, \tilde{v})-12\left(\int_{0}^{1} d u C(u, \tilde{v})\right)^{2}}}
$$

\section{E Tail dependence generated by the Student's factor model}

We consider two random variables $X$ and $Y$, related by the relation

$$
X=\alpha Y+\epsilon,
$$


where $\epsilon$ is a random variable independent of $Y$ and $\alpha$ a non random positive coefficient. Assume that $Y$ and $\epsilon$ have a Student's distribution with density:

$$
\begin{gathered}
P_{Y}(y)=\frac{C_{\nu}}{\left(1+\frac{y^{2}}{\nu}\right)^{\frac{\nu+1}{2}}}, \\
P_{\epsilon}(\epsilon)=\frac{C_{\nu}}{\sigma\left(1+\frac{\epsilon^{2}}{\nu \sigma^{2}}\right)^{\frac{\nu+1}{2}}} .
\end{gathered}
$$

We first give a general expression for the probability for $X$ to be larger than $F_{X}^{-1}(u)$ knowing that $Y$ is larger than $F_{Y}^{-1}(u)$ :

LEMMA 1

The probability that $X$ is larger than $F_{X}^{-1}(u)$ knowing that $Y$ is larger than $F_{Y}^{-1}(u)$ is given by:

$$
\operatorname{Pr}\left[X>F_{X}^{-1}(u) \mid Y>F_{Y}^{-1}(u)\right]=\bar{F}_{\epsilon}(\eta)+\frac{\alpha}{1-u} \int_{F_{Y}^{-1}(u)}^{\infty} d y \bar{F}_{Y}(y) \cdot P_{\epsilon}\left[\alpha F_{Y}^{-1}(u)+\eta-\alpha y\right],
$$

with

$$
\eta=F_{X}^{-1}(u)-\alpha F_{Y}^{-1}(u)
$$

The proof of this lemma relies on a simple integration by part and a change of variable, which are detailed in appendix E.1.

Introducing the notation

$$
\tilde{Y}_{u}=F_{Y}^{-1}(u)
$$

we can show that

$$
\eta=\alpha\left[\left(1+\left(\frac{\sigma}{\alpha}\right)^{\nu}\right)^{1 / \nu}-1\right] \tilde{Y}_{u}+\mathcal{O}\left(\tilde{Y}_{u}^{-1}\right),
$$

which allows us to conclude that $\eta$ goes to infinity as $u$ goes to 1 (see appendix E.2 for the derivation of this result). Thus, $\bar{F}_{\epsilon}(\eta)$ goes to zero as u goes to 1 and

$$
\lambda=\lim _{u \rightarrow 1} \frac{\alpha}{1-u} \int_{\tilde{Y}_{u}}^{\infty} d y \bar{F}_{Y}(y) \cdot P_{\epsilon}\left(\alpha \tilde{Y}_{u}+\eta-\alpha y\right) .
$$

Now, using the following result :

LEMMA 2

Assuming $\nu>0$ and $x_{0}>1$,

$$
\lim _{\epsilon \rightarrow 0} \frac{1}{\epsilon} \int_{1}^{\infty} d x \frac{1}{x^{\nu}} \frac{C_{\nu}}{\left[1+\left(\frac{x-x_{0}}{\epsilon}\right)^{2}\right]^{\frac{\nu+1}{2}}}=\frac{1}{x_{0}^{\nu}}
$$

whose proof is given in appendix E.3, it is straigthforward to show that

$$
\lambda=\frac{1}{1+\left(\frac{\sigma}{\alpha}\right)^{\nu}} .
$$

The final steps of this calculation are given in appendix E.4. 


\section{E.1 Proof of Lemma 1}

By definition,

$$
\begin{aligned}
\operatorname{Pr}\left[X>F_{X}^{-1}(u), Y>F_{Y}^{-1}(u)\right] & =\int_{F_{X}^{-1}(u)}^{\infty} d x \int_{F_{Y}^{-1}(u)}^{\infty} d y P_{Y}(y) \cdot P_{\epsilon}(x-\alpha y) \\
& =\int_{F_{Y}^{-1}(u)}^{\infty} d y P_{Y}(y) \cdot \bar{F}_{\epsilon}\left[F_{X}^{-1}(u)-\alpha y\right]
\end{aligned}
$$

Let us perform an integration by part :

$$
\begin{aligned}
\operatorname{Pr}\left[X>F_{X}^{-1}(u), Y>F_{Y}^{-1}(u)\right] & =\left[-\bar{F}_{Y}(y) \cdot \bar{F}_{\epsilon}\left(F_{X}^{-1}(u)-\alpha y\right)\right]_{F_{Y}^{-1}(u)}^{\infty}+ \\
& +\alpha \int_{F_{Y}^{-1}(u)}^{\infty} d y \bar{F}_{Y}(y) \cdot P_{\epsilon}\left(F_{X}^{-1}(u)-\alpha y\right) \\
& =(1-u) \bar{F}_{\epsilon}\left(F_{X}^{-1}(u)-\alpha F_{Y}^{-1}(u)\right)+ \\
& +\alpha \int_{F_{Y}^{-1}(u)}^{\infty} d y \bar{F}_{Y}(y) \cdot P_{\epsilon}\left(F_{X}^{-1}(u)-\alpha y\right)
\end{aligned}
$$

Defining $\eta=F_{X}^{-1}(u)-\alpha F_{Y}^{-1}(u)$ (see equation(138), and dividing each term by

$$
\operatorname{Pr}\left[Y>F_{Y}^{-1}(u)\right]=1-u
$$

we obtain the result given in (137)

\section{E.2 Derivation of equation (140)}

The factor $Y$ and the idiosyncratic noise $\epsilon$ have Student's distributions with $\nu$ degrees of freedom given by (135) and (136) respectively. It follows that the survival distributions of $Y$ and $\epsilon$ are :

$$
\begin{gathered}
\bar{F}_{Y}(y)=\frac{\nu^{\frac{\nu-1}{2}} C_{\nu}}{y^{\nu}}+\mathcal{O}\left(y^{-(\nu+2)}\right), \\
\bar{F}_{\epsilon}(\epsilon)=\frac{\sigma^{\nu} \nu^{\frac{\nu-1}{2}} C_{\nu}}{\epsilon^{\nu}}+\mathcal{O}\left(\epsilon^{-(\nu+2)}\right),
\end{gathered}
$$

and

$$
\bar{F}_{X}(x)=\frac{\left(\alpha^{\nu}+\sigma^{\nu}\right) \nu^{\frac{\nu-1}{2}} C_{\nu}}{x^{\nu}}+\mathcal{O}\left(x^{-(\nu+2)}\right)
$$

Using the notation (139), equation (138) can be rewritten as

$$
\bar{F}_{X}\left(\eta+\alpha \tilde{Y}_{u}\right)=\bar{F}_{Y}\left(\tilde{Y}_{u}\right)=1-u,
$$

whose solution for large $\tilde{Y}_{u}$ (or equivalently as u goes to 1) is

$$
\eta=\alpha\left[\left(1+\left(\frac{\sigma}{\alpha}\right)^{\nu}\right)^{1 / \nu}-1\right] \tilde{Y}_{u}+\mathcal{O}\left(\tilde{Y}_{u}^{-1}\right) .
$$

To obain this equation, we have used the asymptotic expressions of $\bar{F}_{X}$ and $\bar{F}_{Y}$ given in (152) and (149). 


\section{E.3 Proof of lemma 2}

We want to prove that, assuming $\nu>0$ and $x_{0}>1$,

$$
\lim _{\epsilon \rightarrow 0} \frac{1}{\epsilon} \int_{1}^{\infty} d x \frac{1}{x^{\nu}} \frac{C_{\nu}}{\left[1+\frac{1}{\nu}\left(\frac{x-x_{0}}{\epsilon}\right)^{2}\right]^{\frac{\nu+1}{2}}}=\frac{1}{x_{0}^{\nu}}
$$

The change of variable

$$
u=\frac{x-x_{0}}{\epsilon},
$$

gives

$$
\begin{aligned}
\frac{1}{\epsilon} \int_{1}^{\infty} d x \frac{1}{x^{\nu}} \frac{C_{\nu}}{\left[1+\frac{1}{\nu}\left(\frac{x-x_{0}}{\epsilon}\right)^{2}\right]^{\frac{\nu+1}{2}}} & =\int_{\frac{1-x_{0}}{\epsilon}}^{\infty} d u \frac{1}{\left(\epsilon u+x_{0}\right)^{\nu}} \frac{C_{\nu}}{\left(1+\frac{u^{2}}{\nu}\right)^{\frac{\nu+1}{2}}} \\
& =\frac{1}{x_{0}^{\nu}} \int_{\frac{1-x_{0}}{\epsilon}}^{\infty} d u \frac{1}{\left(1+\frac{\epsilon u}{x_{0}}\right)^{\nu}} \frac{C_{\nu}}{\left(1+\frac{u^{2}}{\nu}\right)^{\frac{\nu+1}{2}}} \\
& =\frac{1}{x_{0}^{\nu}} \int_{\frac{1-x_{0}}{\epsilon}}^{\frac{x_{0}}{\epsilon}} d u \frac{1}{\left(1+\frac{\epsilon u}{x_{0}}\right)^{\nu}} \frac{C_{\nu}}{\left(1+\frac{u^{2}}{\nu}\right)^{\frac{\nu+1}{2}}}+ \\
& +\frac{1}{x_{0}^{\nu}} \int_{\frac{x_{0}}{\epsilon}}^{\infty} d u \frac{1}{\left(1+\frac{\epsilon u}{x_{0}}\right)^{\nu}} \frac{C_{\nu}}{\left(1+\frac{u^{2}}{\nu}\right)^{\frac{\nu+1}{2}}} .
\end{aligned}
$$

Consider the second integral. We have

$$
u \geq \frac{x_{0}}{\epsilon},
$$

which allows us to write

$$
\frac{1}{\left(1+u^{2}\right)^{\frac{\nu+1}{2}}} \leq \frac{\nu^{\frac{\nu+1}{2}} \epsilon^{\nu+1}}{x_{0}^{\nu+1}}
$$

so that

$$
\begin{aligned}
\left|\int_{\frac{x_{0}}{\epsilon}}^{\infty} d u \frac{1}{\left(1+\frac{\epsilon u}{x_{0}}\right)^{\nu}} \frac{C_{\nu}}{\left(1+u^{2}\right)^{\frac{\nu+1}{2}}}\right| & \leq \frac{\nu^{\frac{\nu+1}{2}} \epsilon^{\nu+1}}{x_{0}^{\nu+1}} \int_{\frac{x_{0}}{\epsilon}}^{\infty} d u \frac{C_{\nu}}{\left(1+\frac{\epsilon u}{x_{0}}\right)^{\nu}} \\
& =\frac{\nu^{\frac{\nu+1}{2}} \epsilon^{\nu}}{x_{0}^{\nu}} \int_{1}^{\infty} d v \frac{C_{\nu}}{(1+v)^{\nu}} \\
& =\mathcal{O}\left(\epsilon^{\nu}\right) .
\end{aligned}
$$

The next step of the proof is to show that

$$
\int_{\frac{1-x_{0}}{\epsilon}}^{\frac{x_{0}}{\epsilon}} d u \frac{1}{\left(1+\frac{\epsilon u}{x_{0}}\right)^{\nu}} \frac{C_{\nu}}{\left(1+\frac{u^{2}}{\nu}\right)^{\frac{\nu+1}{2}}} \longrightarrow 1 \text { as } \epsilon \longrightarrow 0 .
$$

Let us calculate

$$
\left|\int_{\frac{1-x_{0}}{\epsilon}}^{\frac{x_{0}}{\epsilon}} d u \frac{1}{\left(1+\frac{\epsilon u}{x_{0}}\right)^{\nu}} \frac{C_{\nu}}{\left(1+\frac{u^{2}}{\nu}\right)^{\frac{\nu+1}{2}}}-1\right|=\mid \int_{\frac{1-x_{0}}{\epsilon}}^{\frac{x_{0}}{\epsilon}} d u \frac{1}{\left(1+\frac{\epsilon u}{x_{0}}\right)^{\nu}} \frac{C_{\nu}}{\left(1+\frac{u^{2}}{\nu}\right)^{\frac{\nu+1}{2}}}-
$$




$$
\begin{aligned}
& -\int_{-\infty}^{\infty} d u \frac{C_{\nu}}{\left(1+\frac{u^{2}}{\nu}\right)^{\frac{\nu+1}{2}}} \mid \\
& =\mid \int_{\frac{1-x_{0}}{\epsilon}}^{\frac{x_{0}}{\epsilon}} d u\left[\frac{1}{\left(1+\frac{\epsilon u}{x_{0}}\right)^{\nu}}-1\right] \frac{C_{\nu}}{\left(1+\frac{u^{2}}{\nu}\right)^{\frac{\nu+1}{2}}}- \\
& -\quad \int_{-\infty}^{\frac{1-x_{0}}{\epsilon}} d u \frac{C_{\nu}}{\left(1+\frac{u^{2}}{\nu}\right)^{\frac{\nu+1}{2}}}-\int_{\frac{x_{0}}{\epsilon}}^{\infty} d u \frac{C_{\nu}}{\left(1+\frac{u^{2}}{\nu}\right)^{\frac{\nu+1}{2}}} \mid \\
& \leq\left|\int_{\frac{1-x_{0}}{\epsilon}}^{\frac{x_{0}}{\epsilon}} d u\left[\frac{1}{\left(1+\frac{\epsilon u}{x_{0}}\right)^{\nu}}-1\right] \frac{C_{\nu}}{\left(1+\frac{u^{2}}{\nu}\right)^{\frac{\nu+1}{2}}}\right|+ \\
& +\left|\int_{-\infty}^{\frac{1-x_{0}}{\epsilon}} d u \frac{C_{\nu}}{\left(1+\frac{u^{2}}{\nu}\right)^{\frac{\nu+1}{2}}}\right|+\left|\int_{\frac{x_{0}}{\epsilon}}^{\infty} d u \frac{C_{\nu}}{\left(1+\frac{u^{2}}{\nu}\right)^{\frac{\nu+1}{2}}}\right|
\end{aligned}
$$

The second and third integrals obviously behave like $\mathcal{O}\left(\epsilon^{\nu}\right)$ when $\epsilon$ goes to zero since we have assumed $x_{0}>1$ what ensures that $\frac{1-x_{0}}{\epsilon} \rightarrow-\infty$ and $\frac{x_{0}}{\epsilon} \rightarrow \infty$ when $\epsilon \rightarrow 0$. For the first integral, we have

$$
\left|\int_{\frac{1-x_{0}}{\epsilon}}^{\frac{x_{0}}{\epsilon}} d u\left[\frac{1}{\left(1+\frac{\epsilon u}{x_{0}}\right)^{\nu}}-1\right] \frac{C_{\nu}}{\left(1+\frac{u^{2}}{\nu}\right)^{\frac{\nu+1}{2}}}\right| \leq \int_{\frac{1-x_{0}}{\epsilon}}^{\frac{x_{0}}{\epsilon}} d u\left|\frac{1}{\left(1+\frac{\epsilon u}{x_{0}}\right)^{\nu}}-1\right| \frac{C_{\nu}}{\left(1+\frac{u^{2}}{\nu}\right)^{\frac{\nu+1}{2}}} .
$$

The function

$$
\left|\frac{1}{\left(1+\frac{\epsilon u}{x_{0}}\right)^{\nu}}-1\right|
$$

vanishes at $u=0$, is convex for $u \in\left[\frac{1-x_{0}}{\epsilon}, 0\right]$ and concave for $u \in\left[0, \frac{x_{0}}{\epsilon}\right]$ (see also figure 9), so that there are two constants $A, B>0$ such that

$$
\begin{aligned}
& \left|\frac{1}{\left(1+\frac{\epsilon u}{x_{0}}\right)^{\nu}}-1\right| \leq-\frac{x_{0}^{\nu}-1}{x_{0}-1} \epsilon \cdot u=-A \cdot \epsilon \cdot u, \quad \forall u \in\left[\frac{1-x_{0}}{\epsilon}, 0\right] \\
& \left|\frac{1}{\left(1+\frac{\epsilon u}{x_{0}}\right)^{\nu}}-1\right| \leq \frac{\nu \epsilon}{x_{0}} u=B \cdot \epsilon \cdot u, \quad \forall u \in\left[0, \frac{x_{0}}{\epsilon}\right] .
\end{aligned}
$$

We can thus conclude that

$$
\begin{aligned}
\left|\int_{\frac{1-x_{0}}{\epsilon}}^{\frac{x_{0}}{\epsilon}} d u\left[\frac{1}{\left(1+\frac{\epsilon u}{x_{0}}\right)^{\nu}}-1\right] \frac{C_{\nu}}{\left(1+\frac{u^{2}}{\nu}\right)^{\frac{\nu+1}{2}}}\right| & \leq-A \cdot \epsilon \int_{\frac{1-x_{0}}{\epsilon}}^{0} d u \frac{u \cdot C_{\nu}}{\left(1+\frac{u^{2}}{\nu}\right)^{\frac{\nu+1}{2}}} \\
& +B \cdot \epsilon \int_{0}^{\frac{x_{0}}{\epsilon}} d u \frac{u \cdot C_{\nu}}{\left(1+\frac{u^{2}}{\nu}\right)^{\frac{\nu+1}{2}}} \\
& =\mathcal{O}\left(\epsilon^{\alpha}\right),
\end{aligned}
$$

with $\alpha=\min \{\nu, 1\}$. Indeed, the two integrals can be perfomed exactly, which shows that they behave as $\mathcal{O}(1)$ if $\nu>1$ and as $\mathcal{O}\left(\epsilon^{\nu-1}\right)$ otherwise. Thus, we finally obtain

$$
\left|\int_{\frac{1-x_{0}}{\epsilon}}^{\frac{x_{0}}{\epsilon}} d u \frac{1}{\left(1+\frac{\epsilon u}{x_{0}}\right)^{\nu}} \frac{C_{\nu}}{\left(1+\frac{u^{2}}{\nu}\right)^{\frac{\nu+1}{2}}}-1\right|=\mathcal{O}\left(\epsilon^{\alpha}\right) .
$$


Putting together equations (164) and (175) we obtain

$$
\left|\frac{1}{\epsilon} \int_{1}^{\infty} d x \frac{1}{x^{\nu}} \frac{C_{\nu}}{\left[1+\frac{1}{\nu}\left(\frac{x-x_{0}}{\epsilon}\right)^{2}\right]^{\frac{\nu+1}{2}}}-\frac{1}{x_{0}^{\nu}}\right|=\mathcal{O}\left(\epsilon^{\min \{\nu, 1\}}\right),
$$

which concludes the proof.

\section{E.4 Derivation of equation (143)}

From equation (149, we can deduce

$$
\bar{F}_{Y}(y)=\frac{\nu^{\frac{\nu-1}{2}} C_{\nu}}{y^{\nu}}\left(1+\mathcal{O}\left(y^{-2}\right)\right) .
$$

Using equations (136) and (140), we obtain

$$
P_{\epsilon}\left(\alpha \tilde{Y}_{u}+\eta-\alpha y\right)=P_{\epsilon}\left(\gamma \tilde{Y}_{u}-\alpha y\right) \cdot\left(1+\mathcal{O}\left(\tilde{Y}_{u}^{-2}\right)\right)
$$

where

$$
\gamma=\alpha\left(1+\left(\frac{\sigma}{\alpha}\right)^{\nu}\right)^{1 / \nu}
$$

Putting together these results yields for the leading order

$$
\begin{aligned}
\int_{\tilde{Y}_{u}}^{\infty} d y \bar{F}_{Y}(y) \cdot P_{\epsilon}\left(\alpha \tilde{Y}_{u}+\eta-\alpha y\right) & =\int_{\tilde{Y}_{u}}^{\infty} d y \frac{\nu^{\frac{\nu-1}{2}} C_{\nu}}{y^{\nu}} \cdot \frac{C_{\nu}}{\sigma\left(1+\frac{\left(\gamma \tilde{Y}_{u}-\alpha y\right)^{2}}{\nu \sigma^{2}}\right)^{\frac{\nu+1}{2}}} \\
& =\frac{\nu^{\frac{\nu-1}{2}} C_{\nu}}{\alpha \tilde{Y}_{u}^{\nu}} \int_{1}^{\infty} d x \frac{1}{x^{\nu}} \cdot \frac{C_{\nu} \frac{\alpha \tilde{Y}_{u}}{\sigma}}{\left(1+\frac{1}{\nu}\left(\frac{x-\frac{\gamma}{\alpha}}{\frac{\sigma}{\alpha \tilde{Y}_{u}}}\right)^{2}\right)^{\frac{\nu+1}{2}}}
\end{aligned}
$$

where the change of variable $x=\frac{y}{\tilde{Y}_{u}}$ has been performed in the last equation.

We now apply lemma 2 with $x_{0}=\frac{\gamma}{\alpha}>1$ and $\epsilon=\frac{\sigma}{\alpha \tilde{Y}_{u}}$ which goes to zero as u goes to 1 . This gives

$$
\int_{\tilde{Y}_{u}}^{\infty} d y \bar{F}_{Y}(y) \cdot P_{\epsilon}\left(\alpha \tilde{Y}_{u}+\eta-\alpha y\right) \sim_{u \rightarrow 1} \frac{\nu^{\frac{\nu-1}{2}} C_{\nu}}{\alpha \tilde{Y}_{u}^{\nu}}\left(\frac{\alpha}{\gamma}\right)^{\nu},
$$

which shows that

$$
\operatorname{Pr}\left[X>F_{X}^{-1}(u), Y>F_{Y}^{-1}(u)\right] \sim_{u \rightarrow 1} F_{Y}^{-1}\left(\tilde{Y}_{u}\right)\left(\frac{\alpha}{\gamma}\right)^{\nu}=(1-u)\left(\frac{\alpha}{\gamma}\right)^{\nu},
$$

thus

$$
\operatorname{Pr}\left[X>F_{X}^{-1}(u) \mid Y>F_{Y}^{-1}(u)\right] \sim_{u \rightarrow 1}\left(\frac{\alpha}{\gamma}\right)^{\nu}
$$

which finally yields

$$
\lambda=\frac{1}{1+\left(\frac{\sigma}{\alpha}\right)^{\nu}}
$$




\section{References}

[Abramovitz and Stegun (1972)] Abramovitz, E. and I.A. Stegun, 1972, Handbook of Mathematical functions (Dover Publications, New York).

[Andersen and Sornette (2001)] Andersen, J.V. and D. Sornette, 2001, Have your cake and eat it too: increasing returns while lowering large risks! Journal of Risk Finance 2, 70-82.

[Ang and Bekaert (2000)] Ang, A. and G. Bekaert, 2000, International asset allocation with regime shifts, Working paper.

[Ang and Chen (2001)] Ang, A. and J. Chen, 2001, Asymmetric correlations of equity portfolios, Working Paper.

[Bhansali and Wise (2001)] Bhansali, V. and M.B. Wise, 2001, Forecasting portfolio risk in normal and stressed markets, working paper (preprint at http://xxx.lanl.gov/abs/nlin.AO/0108022)

[Bookstaber (1997)] Bookstaber, R., 1997, Global risk management: are we missing the point? Journal of Portfolio Management, 23, 102-107.

[Boyer et al. (1997)] Boyer, B.H., M.S Gibson and M. Lauretan, 1997, Pitfalls in tests for changes in correlations, International Finance Discussion Paper 597, Board of the Governors of the Federal Reserve System.

[Davis et al. (1999)] Davis, R.A., T. Mikosch and B. Basrak, 1999, Sample ACF of multivariate stochastic recurrence equations with application to $\mathrm{GARCH}$, Working paper.

[Cizeau et al. (2001)] Cizeau, P., M. Potters and J.P. Bouchaud, 2001, Correlation structure of extreme stock returns, Quantitative Finance 1, 217-222.

[Coles et al. (1999)] Coles, S., J. Heffernan and J. Tawn, 1999, Dependence measures for extreme value analyses, Extremes 2, 339-365.

[Embrechts et al. (1999)] Embrechs, P., A.J. McNeil and D. Straumann, 1999, Correlation : Pitfalls and Alternatives. Risk, 69-71.

[Embrechts et al. (2001)] Embrechts, P., A.J. McNeil and D. Straumann, 2001, Correlation and Dependency in Risk Management : Properties and Pitfalls, in : Dempster, M., ed., Value at Risk and Beyond (Cambridge University Press).

[Forbes and Rigobon (2001)] Forbes, K.J. and R. Rigobon, 2001, No contagion, only interdependence: measuring stock market co-movements, forthcoming Journal of Finance.

[Frees and Valdez (1998)] Frees, E. and E. Valdez, 1998, Understanding Relationships using copulas, North Americam Actuarial Journal 2, 1-25.

[Hartmann et al. (2001)] Hartman, P., S. Straetmans and C.G. de Vries, 2001, Asset market linkages in crisis periods, European Central Bank, Working paper $\mathrm{n}^{\circ} 71$.

[Hauksson et al. (2001)] Hauksson, H.A., M.M. Dacorogna, T. Domenig, U.A. Müller and G. Samorodnitsky, 2001, Multivariate Extremes, Aggregation and Risk Estimation, Quantitative Finance 1, 79-95.

[Heffernan (2000)] Herffernan J.E., 2000, A directory of tail dependence, Extremes 3, 279-290. 
[Hult and Lindskog (2001)] Hult, H. and F. Lindskog, 2001, Multivariate extremes, aggregation and dependence in elliptical distributions, Risklab working paper.

[Jensen (1995)] Jensen, J.L., 1995, Saddlepoint Approximations (Oxford University Press).

[Joe (1997)] Joe, H., 1997, Multivariate models and dependence concepts (Chapman \& Hall, London)

[Johnson and Kotz (1972)] Johnson, N.L. and S. Kotz, 1972, Distributions in statistics: Continuous multivariate distributions (John Willey and Sons).

[King and Wadhwani (1990)] King, M. and S. Wadhwani, 1990, Transmission of volatility between stock markets, The Review of Financial Studies 3, 5-330.

[Kulpa (1999)] Kulpa, T., 1999, On approximations of copulas, international Journal of Mathematics and Mathematical sciences 22, 259-269.

[Ledford and Tawn (1996)] Ledford, A.W. and J.A. Tawn,1996 Statistics for near independence in multivariate extrem values, Biometrika 83, 169-187.

[Ledford and Tawn (1998)] Ledford, A.W. and J.A. Tawn, 1998, Concomitant tail behavior for extremes, Adv. Appl. Prob. 30, 197-215.

[Li et al. (1998)] Li, X., P. Mikusincki and M.D. Taylor, 1998, Strong approximation of copulas, Journal of Mathemetical Analisys and Applications 225, 608-623.

[Lindskog (1999)] Lindskog, F., Modelling Dependence with Copulas, Risklab working paper.

[Longin and Solnik (1995)] Longin F. and B. Solnik, 1995, Is the correlation in international equity returns constant: 1960-1990? Journal of International Money and Finance 14, 3-26.

[Longin and Solnik (2001)] Longin F. and B. Solnik, 2001, Extreme Correlation of International Equity Markets, The Journal of Finance LVI, 649-676.

[Loretan (2000)] Loretan, M., 2000, Evaluating changes in correlations during periods of high market volatility, Global Investor 135, 65-68.

[Loretan and English (2000)] Loretan, M. and W.B. English, 2000, Working paper 000-658, Board of Governors of the Federal Reserve System

[Malevergne and Sornette (2001)] Malevergne, Y. and D.Sornette, 2001, Testing the Gaussian copula hypothesis for financial assets dependence, Working paper.

[Malevergne and Sornette (2002)] Malevergne, Y. and D.Sornette, 2002, Tail dependence for factor models, Working paper.y

[Mansilla (2001)] Mansilla, R., 2001, Algorithmic complexity of real financial markets, Physica A 301, 483-492.

[Meerschaert and Scheffler (2001)] Meerschaert, M.M. and H.P. Scheffler, 2001, Sample cross-correlations for moving averages with regularly varying tails, Journal of Time Series Analysis 22, 481-492.

[Nelsen (1998)] Nelsen, R.B., 1998, An Introduction to Copulas. Lectures Notes in statistic 139 (Springer Verlag, New York).

[Patton (2001)] Patton, J.A., 2001, Estimation of copula models for time series of possibly different lengths, U of California, Econ. Disc. Paper No. 2001-17. 
[Quintos (2001)] Quintos, C.E., 2001, Estimating tail dependence and testing for contagion using tail indices, working paper.

[Quintos et al. (2001)] Quintos, C.E., Z.H. Fan and P.C.B. Phillips, 2001, Structural change tests in tail behaviour and the Asian crisis, Review of Economic Studies 68, 633-663.

[Ramchand and Susmel (1998)] Ramchand, L. and R. Susmel, 1998, Volatility and cross correlation across major stock markets, Journal of Empirical Finance 5, 397-416.

[Ross (1976)] Ross, S., 1976, The arbitrage theory of capital asset pricing, Journal of Economic Theory 17, 254-286.

[Scaillet (2000)] Scaillet, O. 2000, Nonparametric estimation of copulas for time series, Working paper.

[Sharpe (1964)] Sharpe, W., 1964, Capital assets prices: a theory of market equilibrium under conditions of risk, Journal of Finance, 19, 425-442.

[Silvapulle and Granger (2001)] Silvapulle, P. and C.W.J. Granger, 2001, Large returns, conditional correlation and portfolio diversification: a value-at-risk approach, Quantitative Finance 1, 542-551.

[Sornette et al. (2000a)] Sornette, D. P. Simonetti and J. V. Andersen, 2000, $\phi^{q}$-field theory for Portfolio optimization: "fat tails" and non-linear correlations, Physics Report 335, 19-92.

[Sornette et al. (2000b)] Sornette, D., J.V. Andersen and P. Simonetti, 2000, Portfolio Theory for "Fat Tails", International Journal of Theoretical and Applied Finance 3, 523-535.

[Starica (1999)] Starica, C., 1999, Multivariate extremes for models with constant conditional correlations, Journal of Empirical Finance 6, 515-553.

[Tsui and Yu (1999)] Tsui, A.K. and Q. Yu, 1999, Constant conditional correlation in a bivariate GARCH model: evidence from the stock markets of China, Mathematics and Computers in Simulation 48, 503-509. 


\begin{tabular}{|c|c|c|c|}
\hline & $\rho_{v}^{+}$ & $\rho_{v}^{s}$ & $\rho_{u}$ \\
\hline Bivariate Gaussian & $\frac{\rho}{\sqrt{1-\rho^{2}}} \cdot \frac{1}{v}(\rrbracket)$ & $1-\frac{1}{2} \frac{1-\rho^{2}}{\rho^{2}} \frac{1}{v^{2}}($ (6) & $\rho \frac{1+\rho}{1-\rho} \cdot \frac{1}{u^{2}}(17)$ \\
\hline Bivariate Student's & $\frac{\rho}{\sqrt{\rho^{2}+(\nu-1) \sqrt{\frac{\nu-2}{\nu}}\left(1-\rho^{2}\right)}}(8)$ & $\frac{\rho}{\sqrt{\rho^{2}+\frac{1}{(\nu-1)} \sqrt{\frac{\nu-2}{\nu}}\left(1-\rho^{2}\right)}}$ (9) & - \\
\hline Gaussian Factor Model & same as (\#) & same as (6) & same as (17) \\
\hline Student's Factor Model & $1-\frac{K}{v^{2}}(13)$ & $1-\frac{K}{v^{2}}(13)$ & - \\
\hline
\end{tabular}

Table 1: Large $v$ and $u$ dependence of the conditional correlations $\rho_{v}^{+}$(signed condition), $\rho_{v}^{s}$ (unsigned condition) and $\rho_{u}$ (on both variables) for the different models studied in the present paper, described in the first column. The numbers in parentheses give the equation numbers from which the formulas are derived. The factor model is defined by (10), i.e., $X=\alpha Y+\epsilon . \rho$ is the unconditional correlation coefficient.

\begin{tabular}{||c|c|c|c|c|c||}
\hline \hline & $\rho_{v=\infty}^{+}$ & $\rho_{v=\infty}^{s}$ & $\rho_{u=\infty}$ & $\lambda$ & $\lambda$ \\
\hline Bivariate Gaussian & 0 & 1 & 0 & 0 & $\rho$ \\
\hline Bivariate Student's & see Table 1 1 & see Table 1 & - & $2 \cdot \bar{T}_{\nu+1}\left(\sqrt{\nu+1} \sqrt{\frac{1-\rho}{1+\rho}}\right)$ & 1 \\
\hline Gaussian Factor Model & 0 & 1 & 0 & 0 & $\rho$ \\
\hline Student's Factor Model & 1 & 1 & - & $\frac{\rho^{\nu}}{\rho^{\nu}+\left(1-\rho^{2}\right)^{\nu / 2}}$ & 1 \\
\hline \hline
\end{tabular}

Table 2: Asymptotic values of $\rho_{v}^{+}, \rho_{v}^{s}$ and $\rho_{u}$ for $v \rightarrow+\infty$ and $u \rightarrow \infty$ and comparison with the taildependence $\lambda$ and $\bar{\lambda}$ for the four models indicated in the first column. The factor model is defined by (10), i.e., $X=\alpha Y+\epsilon . \rho$ is the unconditional correlation coefficient. For the Student's factor model, $Y$ and $\epsilon$ have centered Student's distributions with the same number $\nu$ of degrees of freedom and their scale factors are respectively equal to 1 and $\sigma$, so that $\rho=\left(1+\frac{\sigma^{2}}{\alpha^{2}}\right)^{-1 / 2}$. For the Bivariate Student's distribution, we refer to Table 1 for the constant values of $\rho_{v=\infty}^{+}$and $\rho_{v=\infty}^{s}$. 

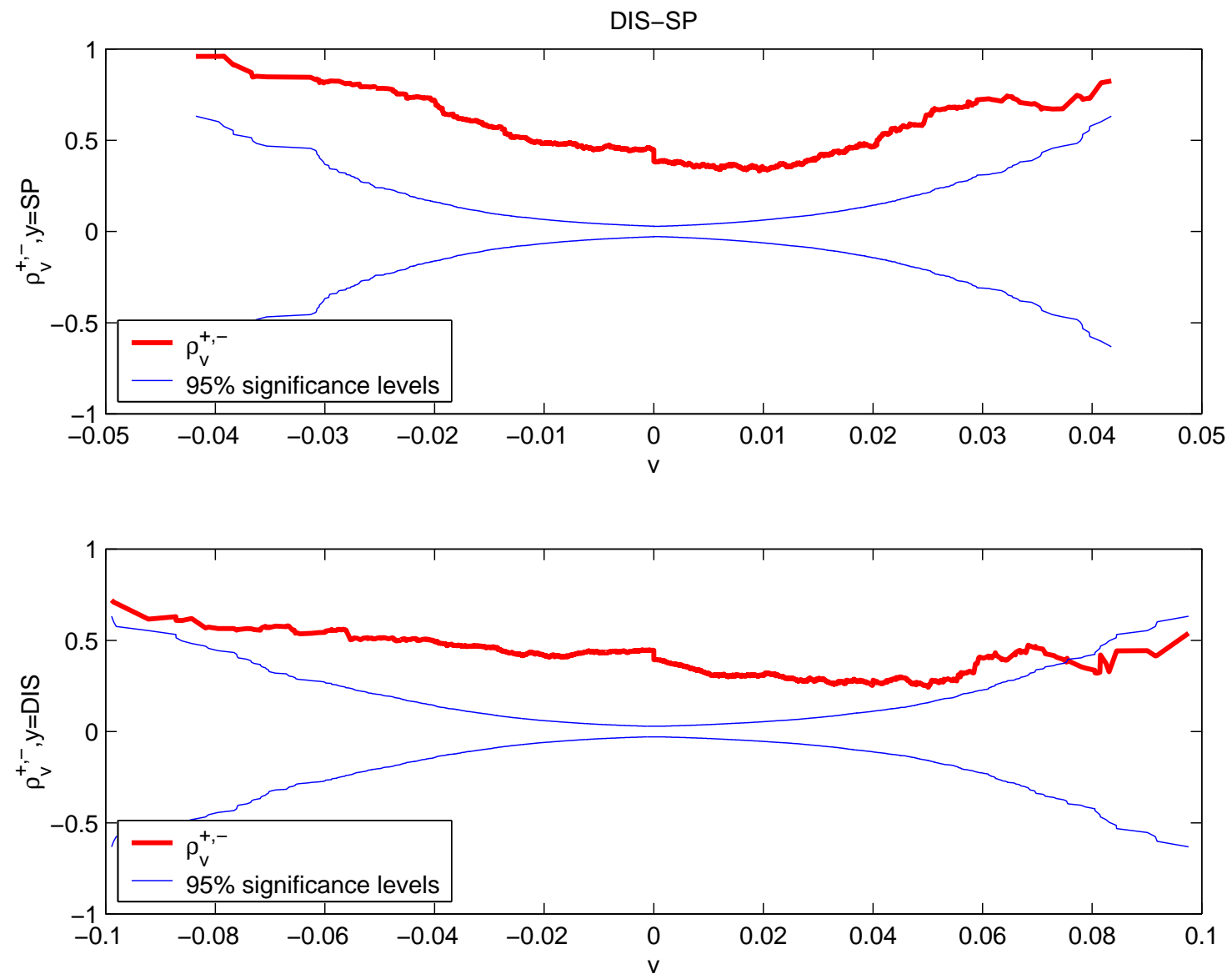

Figure 1: In the upper panel, the thick curve depicts the correlation coefficient between the Disney Company daily returns and the Standard \& Poor's 500 index daily returns conditional on the Standard \& Poor's 500 index daily returns larger than (smaller than) a given positive (negative) value $v$. The two thin curves represent the area within which we cannot consider, at the $95 \%$ confidence level, that the estimated correlation coefficient is significantly different from zero. The lower panel gives the same kind of information but for the correlation coefficient conditioned on the Disney Company daily returns larger than (smaller than) a given positive (negative) value $v$. 

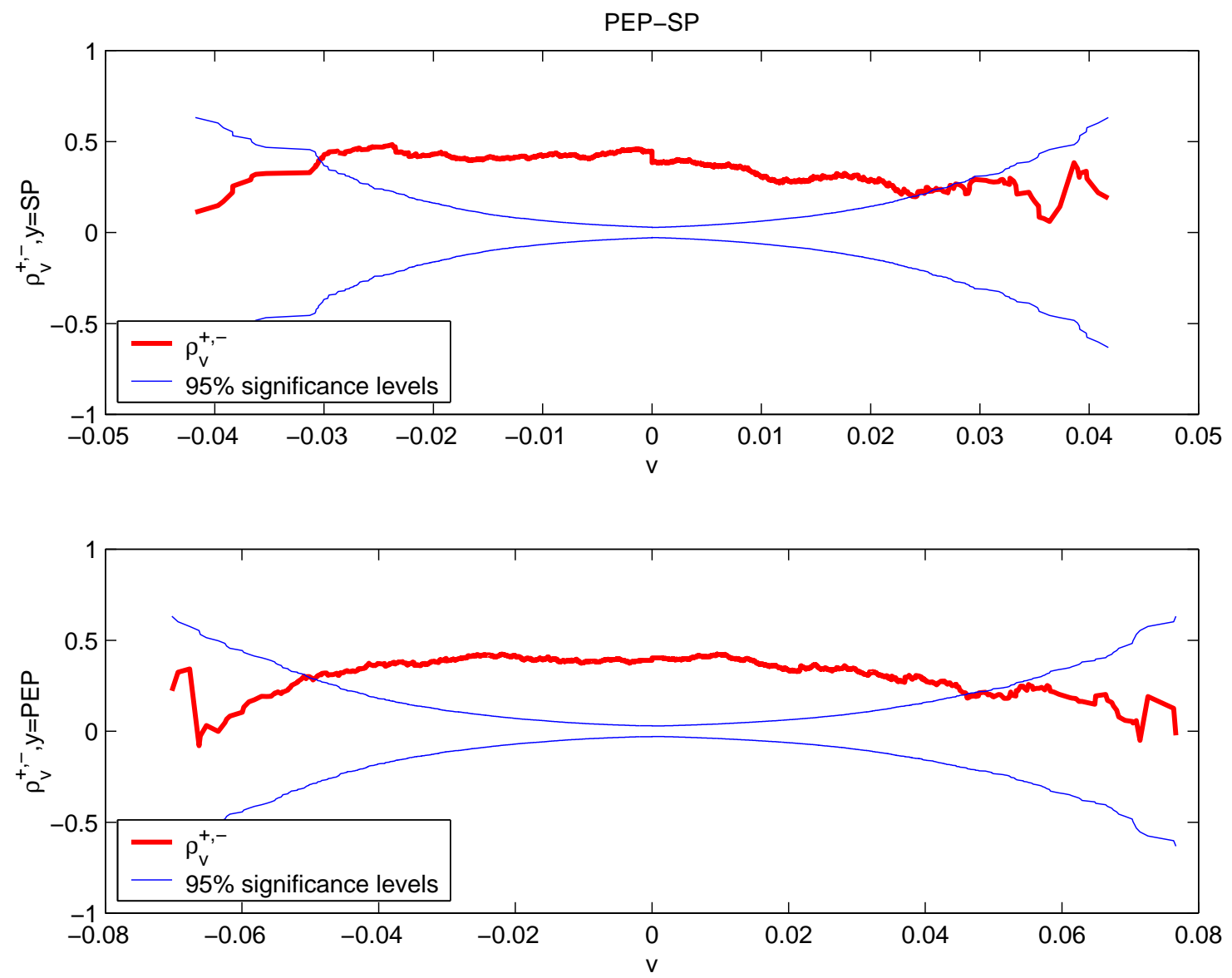

Figure 2: In the upper panel, the thick curve depicts the correlation coefficient between the Pepsico Incorporated daily returns and the Standard \& Poor's 500 index daily returns conditional on the Standard \& Poor's 500 index daily returns larger than (smaller than) a given positive (negative) value $v$. The two thin curves represent the area within which we cannot consider, at the $95 \%$ confidence level, that the estimated correlation coefficient is significantly different from zero. The lower panel gives the same kind of information but for the correlation coefficient conditioned on the Pepsico Incorporated daily returns larger than (smaller than) a given positive (negative) value $v$. 


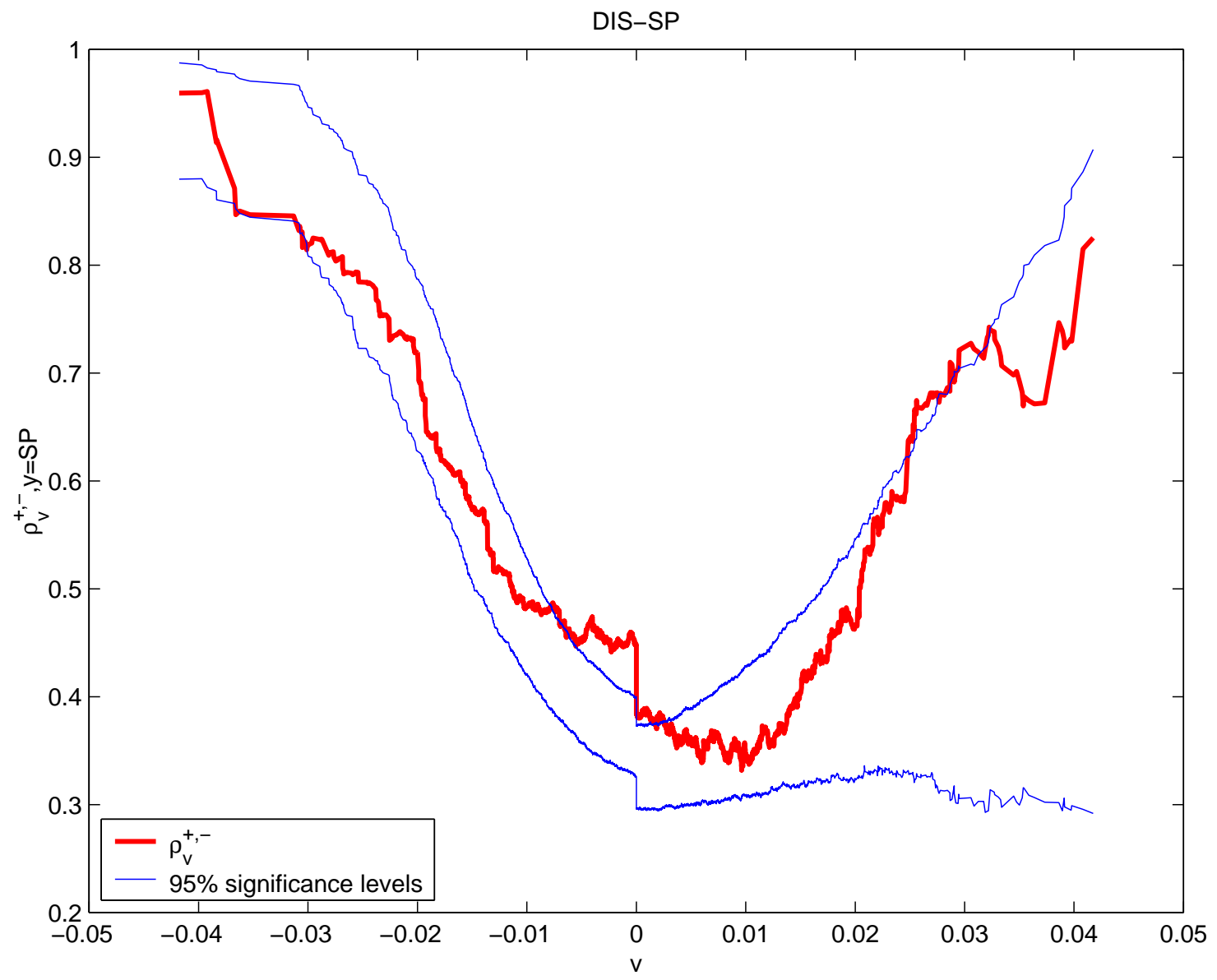

Figure 3: The thick curve depicts the correlation coefficient between the Disney Company daily returns and the Standard \& Poor's 500 index daily returns conditional on the Standard \& Poor's 500 index daily returns larger than (smaller than) a given positive (negative) value $v$. The two thin curves represent the borders within which this conditional correlation coefficient must lay in order to comply, at the $95 \%$ confidence level, with the assumption according to which the Disney Company daily returns can be explained by a one factor model, whose factor is given by the Standard \& Poor's 500 index daily returns. 


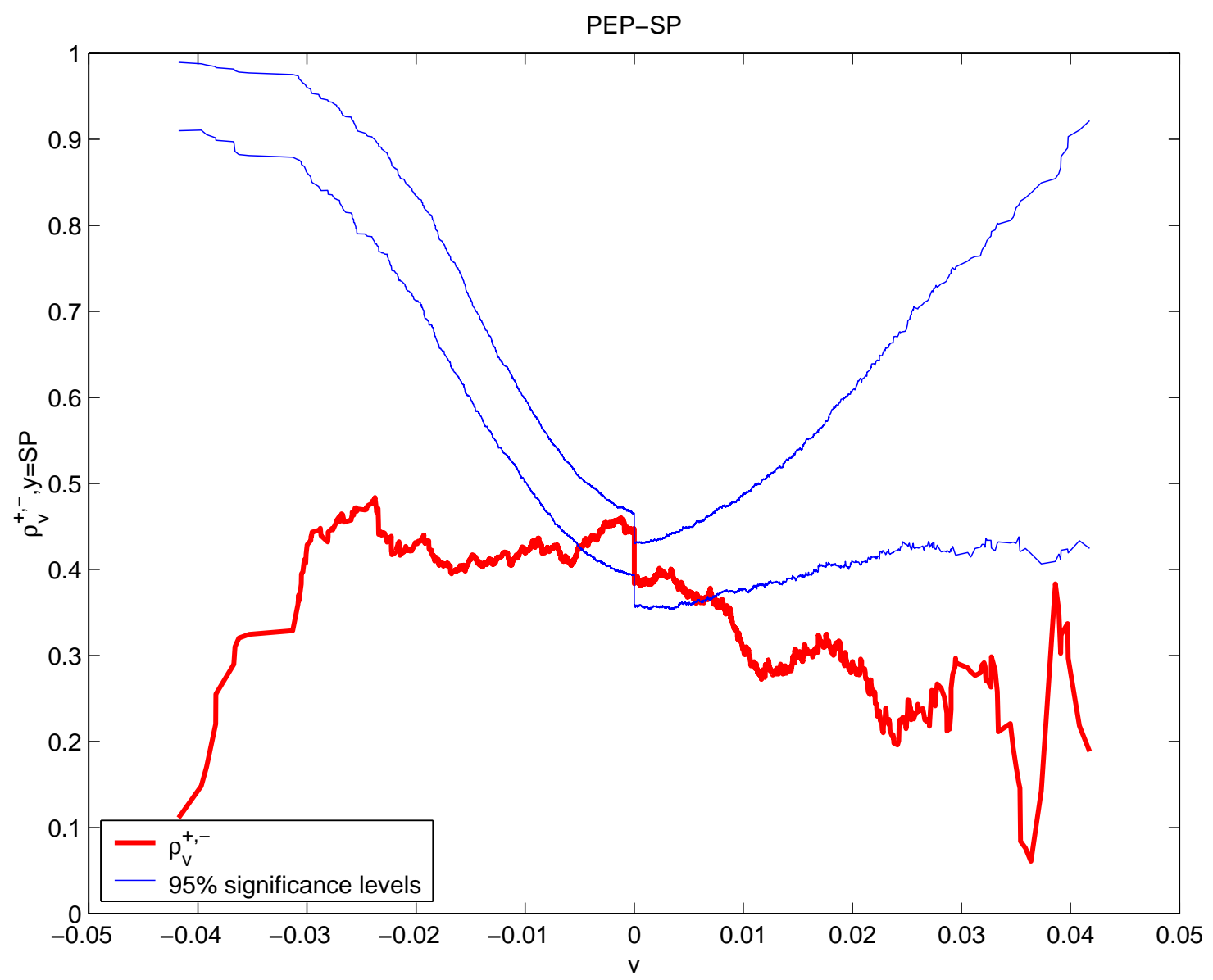

Figure 4: the thick curve depicts the correlation coefficient between the Pepsico Incorporated daily returns and the Standard \& Poor's 500 index daily returns conditional on the Standard \& Poor's 500 index daily returns larger than (smaller than) a given positive (negative) value $v$. The two thin curves represent the borders within which this conditional correlation coefficient must lay in order to comply, at the $95 \%$ confidence level, with the assumption according to which the Disney Company daily returns can be explained by a one factor model, whose factor is given by the Standard \& Poor's 500 index daily returns. 


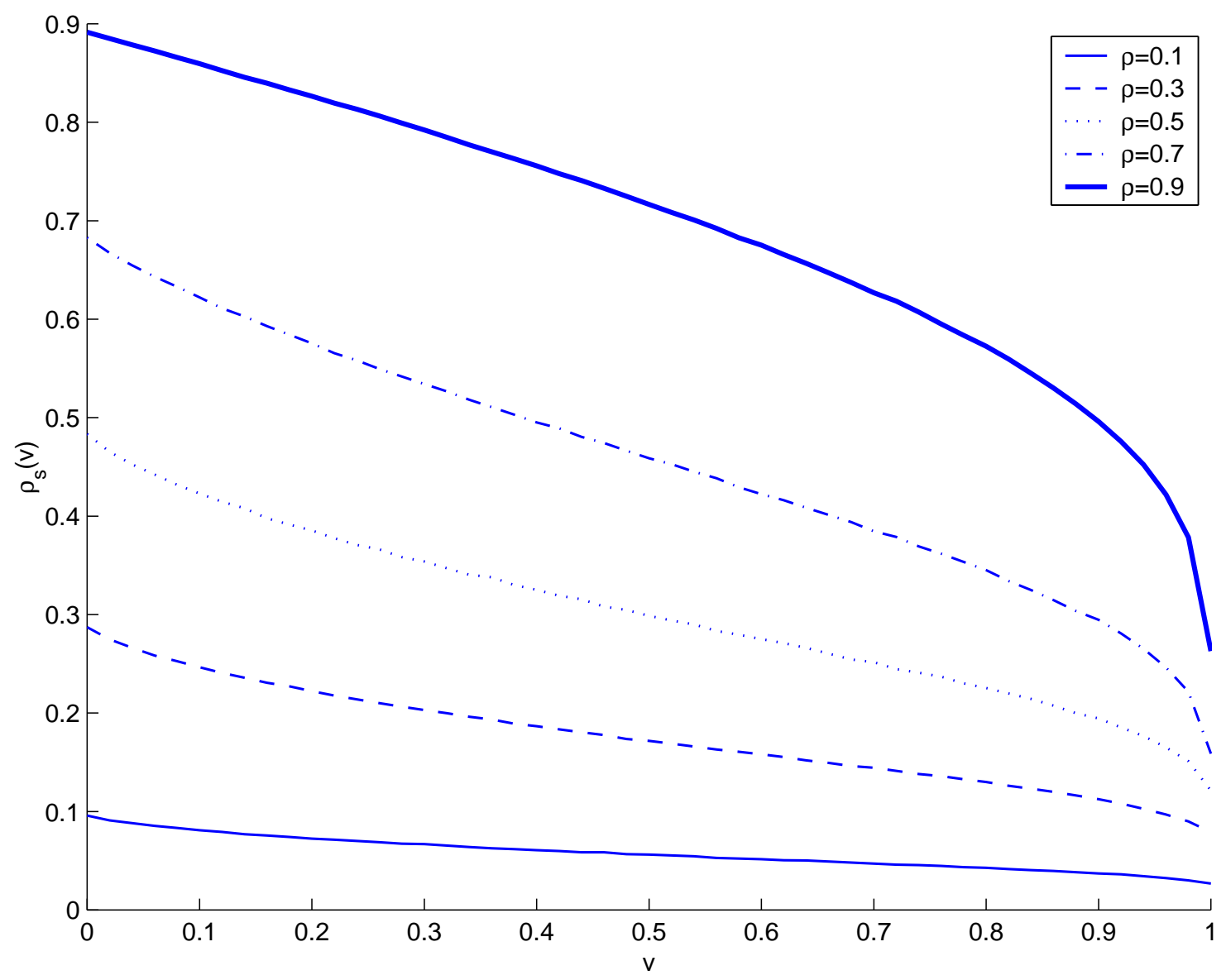

Figure 5: Conditional Spearman's rho for bivatriate Gaussian distributions with unconditional linear correlation coefficient $\rho=0.1,0.3,0.5,0.7,0.9$. 

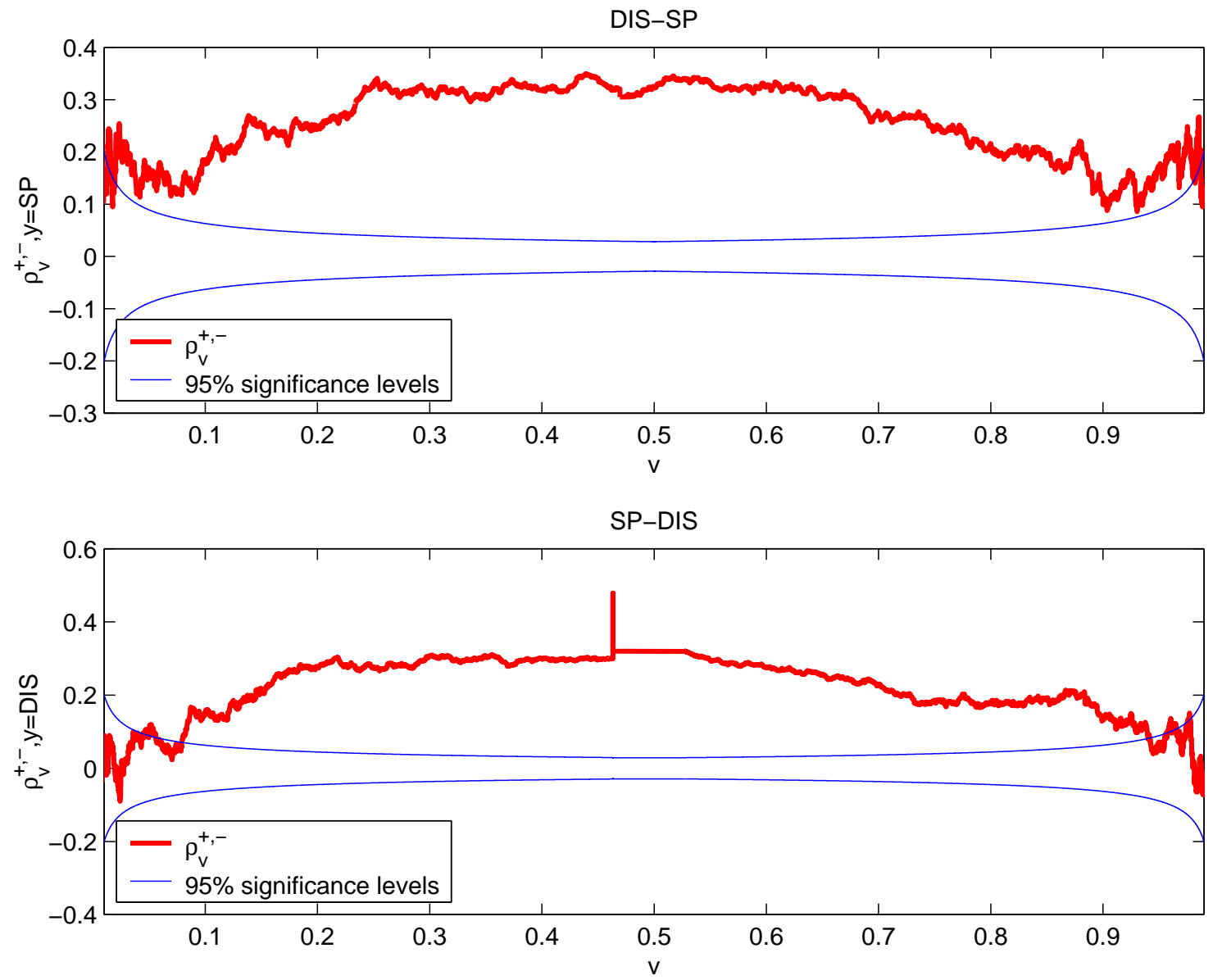

Figure 6: In the upper panel, the thick curve depicts the Spearman's rho between the Disney Company daily returns and the Standard \& Poor's 500 index daily returns. Above the quantile $v=0.5$, the Spearman's rho is conditioned on the Standard \& Poor's 500 index daily returns whose quantile is larger than v, while below the quantile $v=0.5$ it is conditioned on the Standard \& Poor's 500 index daily returns whose quantile is smaller than v. The two thin curves represent the area within which we cannot consider, at the 95\% confidence level, that the estimated Spearman's rho is significantly different from zero. The lower panel gives the same kind of information but for the Spearman's rho conditioned on the realizations of the Disney Company daily returns. 

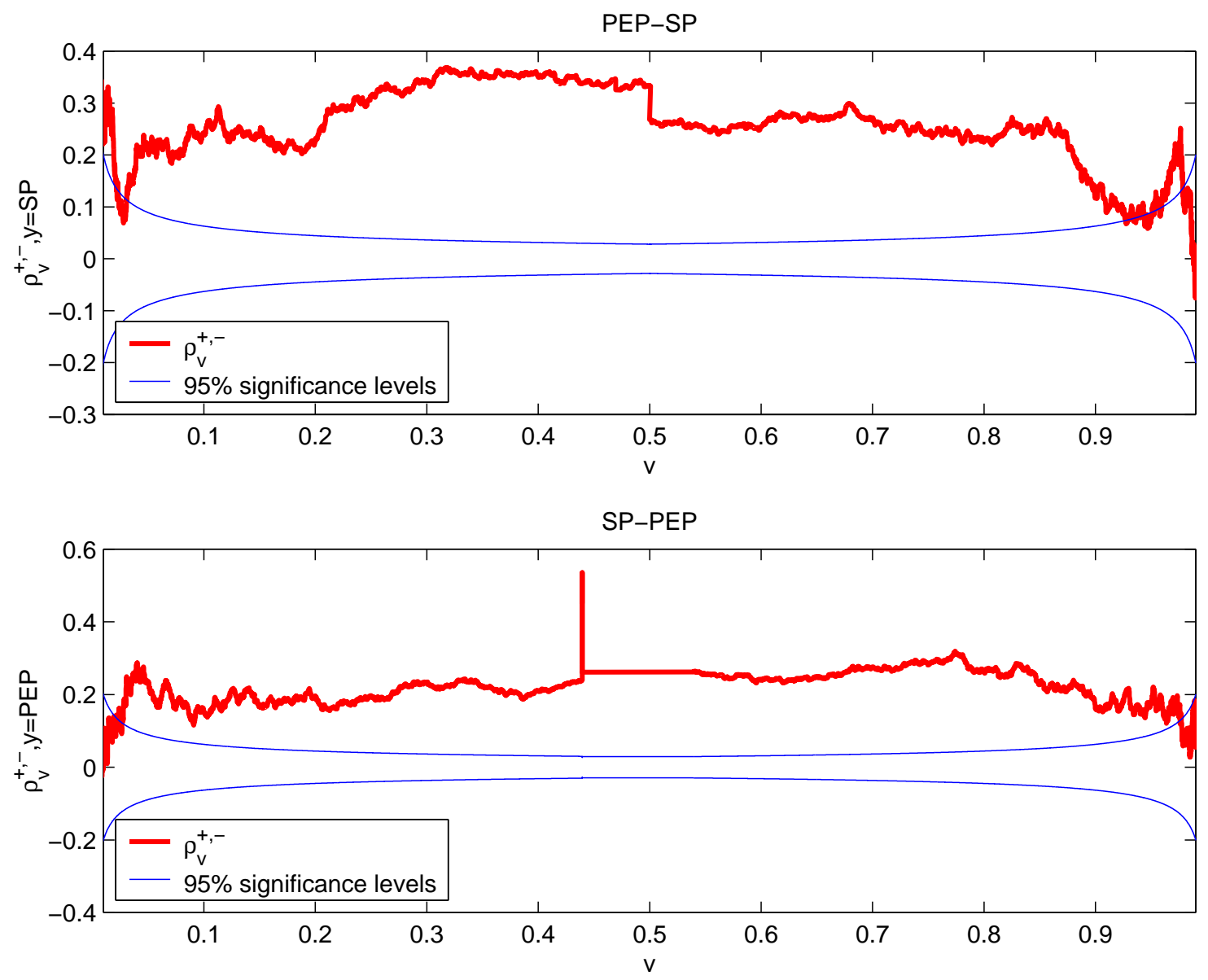

Figure 7: In the upper panel, the thick curve depicts the Spearman's rho between the Pepsico Incorporated daily returns and the Standard \& Poor's 500 index daily returns. Above the quantile $v=0.5$, the Spearman's rho is conditioned on the Standard \& Poor's 500 index daily returns whose quantile is larger than v, while below the quantile $v=0.5$ it is conditioned on the Standard \& Poor's 500 index daily returns whose quantile is smaller than $\mathrm{v}$. The two thin curves represent the area within which we cannot consider, at the 95\% confidence level, that the estimated Spearman's rho is significantly different from zero. The lower panel gives the same kind of information but for the Spearman's rho conditioned on the realizations of the Pepsico Incorporated daily returns. 

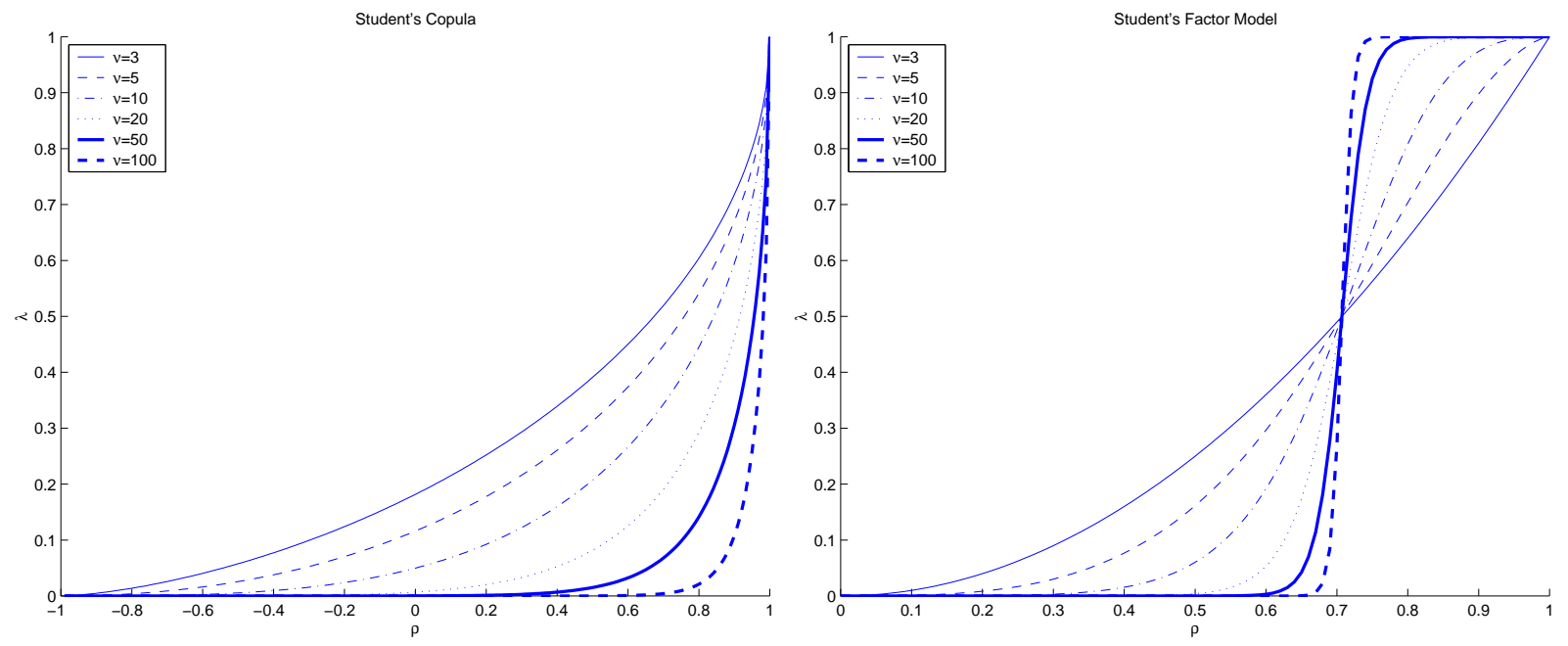

Figure 8: Coefficient of upper tail dependence as a function of the correlation coefficient $\rho$ for various values of the number of degres of freedomn $\nu$ for the student's Copula (left panel) and the Student's factor model (right panel). 


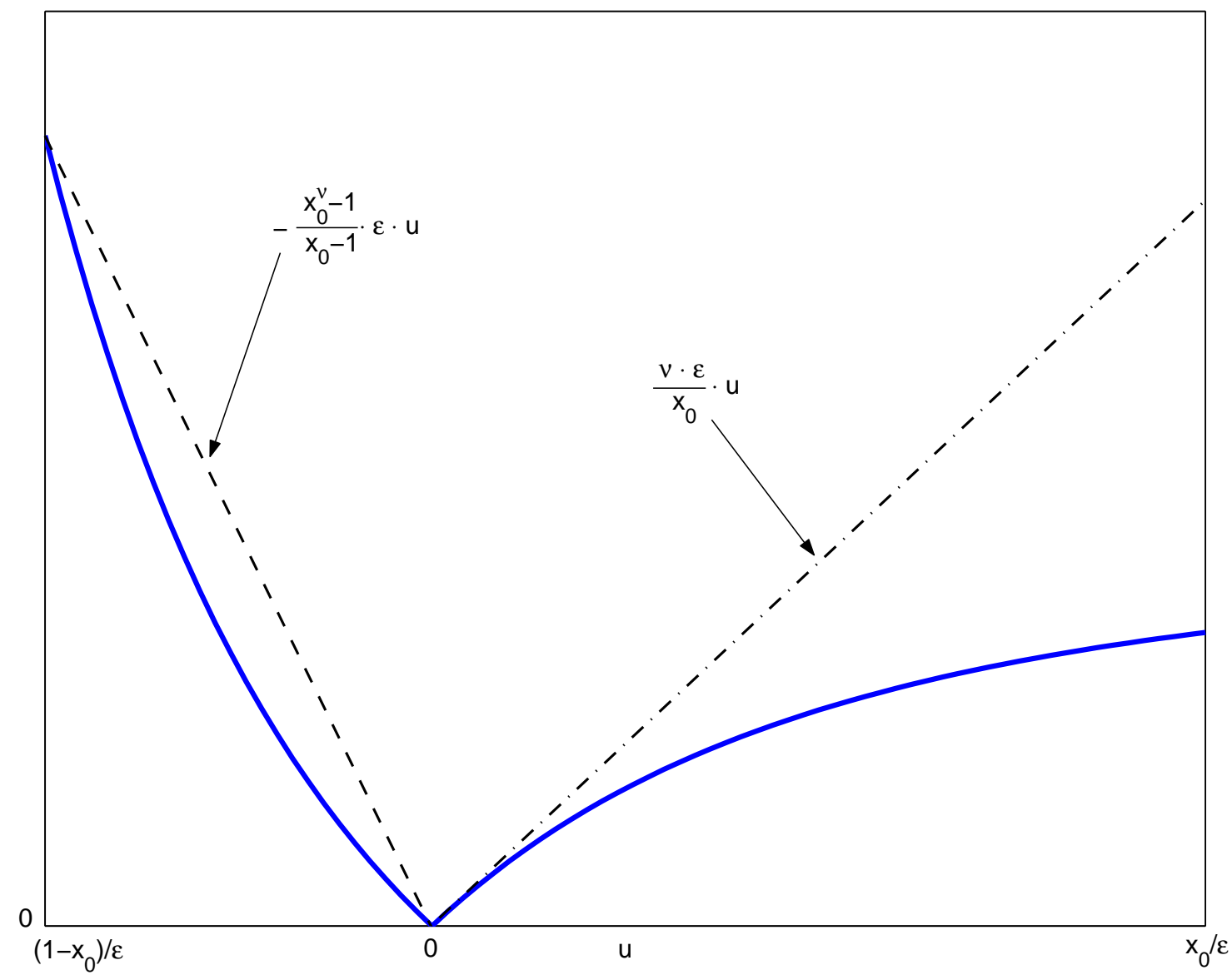

Figure 9: The graph of the function $\left|\frac{1}{\left(1+\frac{\epsilon u}{x_{0}}\right)^{\nu}}-1\right|$ (thick solid line), the string which gives an upper bound of the function within $\left[\frac{1-x_{0}}{\epsilon}, 0\right]$ (dashed line) and the tangent in $0^{+}$which gives an upper bound of the function within $\left[0, \frac{x_{0}}{\epsilon}\right]$ (dash dotted line). 